\title{
Large Deviations in Discrete-Time Renewal Theory
}

\author{
Marco Zamparo*
}

\begin{abstract}
We establish sharp large deviation principles for cumulative rewards associated with a discrete-time renewal model, supposing that each renewal involves a broad-sense reward taking values in a real separable Banach space. The framework we consider is the pinning model of polymers, which amounts to a Gibbs change of measure of a classical renewal process and includes it as a special case. We first tackle the problem in a constrained pinning model, where one of the renewals occurs at a given time, by an argument based on convexity and super-additivity. We then transfer the results to the original pinning model by resorting to conditioning.
\end{abstract}

Keywords: Large deviations; Cramér's theorem; Renewal processes; Polymer pinning models; Renewal-reward processes; Banach space valued random variables

Mathematics Subject Classification 2020: 60F10; 60K05; 60K35

\section{Introduction}

\subsection{Renewals and Cramér's theorem}

Renewal models are widespread tools of probability, finding application in Queueing Theory 1], Insurance [2, and Finance 3] among others. A renewal model describes some event that occurs at the renewal times $T_{1}, T_{2}, \ldots$ involving the rewards $X_{1}, X_{2}, \ldots$ respectively. If $S_{1}, S_{2}, \ldots$ are the waiting times for a new occurrence of the event, then the renewal time $T_{i}$ can be expressed for each $i \geq 1$ in terms of waiting times as $T_{i}=S_{1}+\cdots+S_{i}$. This paper deals with cumulative rewards in renewal models with waiting times taking discrete values and rewards taking values in a Banach space. Specifically, we assume that the waiting time and reward pairs $\left(S_{1}, X_{1}\right),\left(S_{2}, X_{2}\right), \ldots$ form an independent and identically distributed sequence of random variables on a probability space $(\Omega, \mathcal{F}, \mathbb{P})$, the waiting times being valued in $\{1,2, \ldots\} \cup\{\infty\}$ and the rewards being valued in a real separable Banach space $(\mathcal{X},\|\cdot\|)$ equipped with the Borel $\sigma$-field $\mathcal{B}(\mathcal{X})$. Any dependence between $X_{i}$ and $S_{i}$ is allowed. The cumulative reward by the integer time $t \geq 0$ is the random variable $W_{t}:=\sum_{i \geq 1} X_{i} \mathbb{1}_{\left\{T_{i} \leq t\right\}}$, which is measurable because $\mathcal{X}$ is separable [4. The stochastic process $t \mapsto W_{t}$ is the so-called renewal-reward process or compound renewal process, which plays an important role in applications [1 3]. The strong law of large numbers can be proved for a renewalreward process under the optimal hypotheses $\mathbb{E}\left[S_{1}\right]<+\infty$ and $\mathbb{E}\left[\left\|X_{1}\right\|\right]<+\infty, \mathbb{E}$ denoting expectation with respect to the law $\mathbb{P}$, by combining the argument of renewal theory [1] with the classical strong law of large numbers of Kolmogorov in separable Banach spaces 4 .

In this paper we characterize the large fluctuations of the cumulative reward $W_{t}$ by establishing large deviation principles that generalize the Cramér's theorem to discrete-time renewal models. Cramér's theorem describes the large fluctuations of non-random sums of random variables, such as the total reward versus the number of renewals $n$ given by $\sum_{i=1}^{n} X_{i}$. It involves the rate function $I_{\mathrm{C}}$ that maps each point $w \in \mathcal{X}$ in the extended real number $I_{\mathrm{C}}(w):=\sup _{\varphi \in \mathcal{X}^{\star}}\left\{\varphi(w)-\ln \mathbb{E}\left[e^{\varphi\left(X_{1}\right)}\right]\right\}$, where $\mathcal{X}^{\star}$ is the topological dual of $\mathcal{X}$.

\footnotetext{
*Dipartimento Scienza Applicata e Tecnologia, Politecnico di Torino, Corso Duca degli Abruzzi 24, Torino, I-10129, Italy

E-mail: marco.zamparo@uniba.it
} 
The following sharp form of Cramér's theorem has been obtained by Bahadur and Zabell [5] through an argument based on convexity and sub-additivity.

Cramér's theorem. The following conclusions hold:

(a) the function $I_{C}$ is lower semicontinuous and proper convex;

(b) if $G \subseteq \mathcal{X}$ is open, then

$$
\liminf _{n \uparrow \infty} \frac{1}{n} \ln \mathbb{P}\left[\frac{1}{n} \sum_{i=1}^{n} X_{i} \in G\right] \geq-\inf _{w \in G}\left\{I_{C}(w)\right\} ;
$$

(c) if $F \subseteq \mathcal{X}$ is compact, open convex, or closed convex, then

$$
\limsup _{n \uparrow \infty} \frac{1}{n} \ln \mathbb{P}\left[\frac{1}{n} \sum_{i=1}^{n} X_{i} \in F\right] \leq-\inf _{w \in F}\left\{I_{C}(w)\right\} .
$$

Furthermore, if $\mathcal{X}$ is finite-dimensional, then this bound is valid for any closed set $F$ provided that $\mathbb{E}\left[e^{\xi\left\|X_{1}\right\|}\right]<+\infty$ for some number $\xi>0$.

Earlier, Donsker and Varadhan [6] proved Cramér's theorem under the stringent exponential moment condition $\mathbb{E}\left[e^{\xi\left\|X_{1}\right\|}\right]<+\infty$ for all $\xi>0$. Importantly, they showed that under this condition the upper bound in part (c) holds for any closed set $F$ even when $\mathcal{X}$ is infinite-dimensional.

Along with the use as stochastic processes, discrete-time renewal models find application in Equilibrium Statistical Physics with a different interpretation of the time coordinate. In particular, they are employed in studying the phenomenon of polymer pinning, whereby a polymer consisting of $t \geq 1$ monomers is pinned by a substrate at the monomers $T_{1}, T_{2}, \ldots$ that represent renewed events along the polymer chain 7,8 . Supposing that the monomer $T_{i}$ contributes an energy $-v\left(S_{i}\right)$ provided that $T_{i} \leq t, v$ being a real function over $\{1,2, \ldots\} \cup$ $\{\infty\}$ called the potential, the state of the polymer is described by the perturbed law $\mathbb{P}_{t}$ defined on the measurable space $(\Omega, \mathcal{F})$ by the Gibbs change of measure

$$
\frac{d \mathbb{P}_{t}}{d \mathbb{P}}:=\frac{e^{H_{t}}}{Z_{t}}
$$

where $H_{t}:=\sum_{i>1} v\left(S_{i}\right) \mathbb{1}_{\left\{T_{i} \leq t\right\}}$ is the Hamiltonian and the normalizing constant $Z_{t}:=$ $\mathbb{E}\left[e^{H_{t}}\right]$ is the partition function. The model $\left(\Omega, \mathcal{F}, \mathbb{P}_{t}\right)$ is called the pinning model $(\mathrm{PM})$ and generalizes the original renewal model corresponding to the potential $v=0$. The theory of large deviations we develop in this paper is framed within the PM supplied with the hypotheses of aperiodicity and extensivity. The waiting time distribution $p:=\mathbb{P}\left[S_{1}=\cdot\right]$ is said to be aperiodic if $\mathbb{P}\left[S_{1}<\infty\right]>0$ and there is no proper sublattice of $\{1,2, \ldots\}$ containing the support of $p$. We point out that a generic $p$ can be made aperiodic when $\mathbb{P}\left[S_{1}<\infty\right]>0$ by simply changing the time unit.

Assumption 1.1. The waiting time distribution $p$ is aperiodic.

We say that the potential $v$ is extensive if there exists a real number $z_{0}$ such that $e^{v(s)} p(s) \leq e^{z_{o} s}$ for all $s$. For instance, any potential $v$ with the property that $\sup _{s \geq 1}\{v(s) / s\}<$ $+\infty$ is extensive. Extensive potentials are the only that serve Equilibrium Statistical Physics, where the partition function $Z_{t} \geq \mathbb{E}\left[e^{H_{t}} \mathbb{1}_{\left\{S_{1}=t\right\}}\right]=e^{v(t)} p(t)$ is expected to grow exponentially in $t$ in order to define the free energy [7, 8 .

Assumption 1.2. The potential $v$ is extensive.

Together with the PM we consider the constrained pinning model (CPM) where the last monomer is always pinned by the substrate [7,8). It corresponds to the law $\mathbb{P}_{t}^{c}$ defined on the measurable space $(\Omega, \mathcal{F})$ through the change of measure

$$
\frac{d \mathbb{P}_{t}^{c}}{d \mathbb{P}}:=\frac{U_{t} e^{H_{t}}}{Z_{t}^{c}},
$$


where $U_{t}:=\sum_{i>1} \mathbb{1}_{\left\{T_{i}=t\right\}}$ is the renewal indicator, which takes value 1 if $t$ is a renewal and value 0 otherwise, and $Z_{t}^{c}:=\mathbb{E}\left[U_{t} e^{H_{t}}\right]$ is the partition function. Our interest in the CPM is twofold. On the one hand, it turns out to be an effective mathematical tool to tackle the PM. Indeed, we can obtain a large deviation principle within the CPM by an argument based on convexity and super-additivity, and then transfer it to the PM by conditioning. The mentioned argument is a generalization of the approach to Cramér's theorem by Bahadur and Zabell [5, which in turn can be traced back to the method of Ruelle [9] and Lanford [10] for proving the existence of various thermodynamic limits. On the other hand, the CPM is a significant framework in itself because it is the mathematical skeleton of the Poland-Scheraga model of DNA denaturation and of some relevant lattice models of Statistical Mechanics, as discussed by the author in Ref. [11 where use of the theory developed in the present paper is made. These models are the cluster model of fluids proposed by Fisher and Felderhof, the model of protein folding introduced independently by Wako and Saitô first and Muñoz and Eaton later, and the model of strained epitaxy considered by Tokar and Dreyssé. The macroscopic observables that enter the thermodynamic description of these systems turn out to be cumulative rewards corresponding to rewards of the order of magnitude of the waiting times [1].

Before introducing our main results, we must say that the CPM is not well-defined a priori. In fact, it may happen with full probability that the time $t$ is not a renewal, so that $Z_{t}^{c}=0$. However, assumption 1.1 resulting in $Z_{t}^{c}>0$ for every sufficiently large $t$ settles the problem at least for all those $t$. To verify this fact, we observe that aperiodicity of $p$ entails that there exist $m$ coprime integers $\sigma_{1}, \ldots, \sigma_{m}$ such that $p\left(\sigma_{l}\right)>0$ for each $l$. The bound $Z_{t}^{c} \geq \mathbb{E}\left[U_{t} e^{H_{t}} \prod_{i=1}^{n} \mathbb{1}_{\left\{S_{i}=s_{i}\right\}}\right]=\prod_{i=1}^{n} e^{v\left(s_{i}\right)} p\left(s_{i}\right)$ if $t=\sum_{i=1}^{n} s_{i}$ yields $Z_{t}^{c}>0$ whenever $t$ is an integer conical combination of $\sigma_{1}, \ldots, \sigma_{m}$. On the other hand, the Frobenius number $t_{c} \geq 0$ associated with $\sigma_{1}, \ldots, \sigma_{m}$ is finite since these integers are coprime and by definition any $t>t_{c}$ can be expressed as an integer conical combination of them. It follows that $Z_{t}^{c}>0$ for all $t>t_{c}$.

\subsection{Statement of main results}

This section reports the main results of the paper. In the sequel, assumptions 1.1 and 1.2 are tacitly supposed to be satisfied and the topological dual $\mathcal{X}^{\star}$ of $\mathcal{X}$ is understood as a Banach space with the norm induced by $\|\cdot\|$. Let $z$ be the function that maps each linear functional $\varphi \in \mathcal{X}^{\star}$ in the extended real number $z(\varphi)$ defined by

$$
z(\varphi):=\inf \left\{\zeta \in \mathbb{R}: \mathbb{E}\left[e^{\varphi\left(X_{1}\right)+v\left(S_{1}\right)-\zeta S_{1}} \mathbb{1}_{\left\{S_{1}<\infty\right\}}\right] \leq 1\right\}
$$

where the infimum over the empty set is customarily interpreted as $+\infty$. The following proposition puts this function into context by relating $z$ to the scaled cumulant generating function of $W_{t}$ within the CPM. According to this proposition, $z(0)$ turns out to be the free energy of the CPM [7,8] and, more in general, $z(\varphi)$ can be regarded as the free energy of a CPM with the (possibly non-extensive) potential $v+\ln \mathbb{E}\left[e^{\varphi\left(X_{1}\right)} \mid S_{1}=\cdot\right]$.

Proposition 1.1. The function $z$ is proper convex and lower semicontinuous. The following limit holds for every $\varphi \in \mathcal{X}^{\star}$ :

$$
\lim _{t \uparrow \infty} \frac{1}{t} \ln \mathbb{E}\left[U_{t} e^{\varphi\left(W_{t}\right)+H_{t}}\right]=z(\varphi) .
$$

Denoting the expectation with respect to the law $\mathbb{P}_{t}^{c}$ by $\mathbb{E}_{t}^{c}$, proposition 1.1 entails that $\lim _{t \uparrow \infty}(1 / t) \ln \mathbb{E}_{t}^{c}\left[e^{\varphi\left(W_{t}\right)}\right]=z(\varphi)-z(0)$ for all $\varphi \in \mathcal{X}^{\star}$, so that $z-z(0)$ is exactly the scaled cumulant generating function of $W_{t}$ within the CPM. We stress that the number $z(0)$ is finite. Indeed, we have $\mathbb{E}\left[e^{v\left(S_{1}\right)-\zeta S_{1}} \mathbb{1}_{\left\{S_{1}<\infty\right\}}\right]>1$ for all sufficiently negative $\zeta$ as $\mathbb{P}\left[S_{1}<\infty\right]>0$ by assumption 1.1 and, at the same time, $\mathbb{E}\left[e^{v\left(S_{1}\right)-\zeta S_{1}} \mathbb{1}_{\left\{S_{1}<\infty\right\}}\right]=\sum_{s \geq 1} e^{v(s)-\zeta s} p(s) \leq 1$ for all $\zeta \geq z_{o}+\ln 2, z_{o}$ being the number introduced by assumption 1.2 . The function $z$ is finite everywhere in the following case, which is relevant for Statistical Mechanics as it comprises the macroscopic observables that enter the thermodynamic description of the system [11]. 
Example 1.1. The function $z$ is finite everywhere if the reward $X_{1}$ is dominated by the waiting time $S_{1}$ in the sense that $\left\|X_{1}\right\| \leq M S_{1}$ with full probability for some constant $M<$ $+\infty$. This follows from the facts that, for any given $\varphi \in \mathcal{X}^{\star}, \mathbb{E}\left[e^{\varphi\left(X_{1}\right)+v\left(S_{1}\right)-\zeta S_{1}} \mathbb{1}_{\left\{S_{1}<\infty\right\}}\right] \geq$ $\mathbb{E}\left[e^{-M\|\varphi\| S_{1}+v\left(S_{1}\right)-\zeta S_{1}} \mathbb{1}_{\left\{S_{1}<\infty\right\}}\right]>1$ for all sufficiently negative $\zeta$, as $\mathbb{P}\left[S_{1}<\infty\right]>0$ by assumption 1.1, and $\mathbb{E}\left[e^{\varphi\left(X_{1}\right)+v\left(S_{1}\right)-\zeta S_{1}} \mathbb{1}_{\left\{S_{1}<\infty\right\}}\right] \leq \sum_{s \geq 1} e^{M\|\varphi\| s+v(s)-\zeta s} p(s) \leq 1$ for all $\zeta \geq z_{o}+M\|\varphi\|+\ln 2$ with $z_{o}$ given by assumption 1.2 ,

We use the function $z$ to construct a rate function. Let $I$ be the Fenchel-Legendre transform of $z-z(0)$, which associates every point $w \in \mathcal{X}$ with the extended real number $I(w)$ given by

$$
I(w):=\sup _{\varphi \in \mathcal{X}^{\star}}\{\varphi(w)-z(\varphi)+z(0)\} .
$$

The following theorem extends Cramér's theorem to the cumulative reward $W_{t}$ with respect to the CPM and constitutes our first main result. It is proved together with proposition 1.1 in Section 2

Theorem 1.1. The following conclusions hold:

(a) the function I is lower semicontinuous and proper convex;

(b) if $G \subseteq \mathcal{X}$ is open, then

$$
\liminf _{t \uparrow \infty} \frac{1}{t} \ln \mathbb{P}_{t}^{c}\left[\frac{W_{t}}{t} \in G\right] \geq-\inf _{w \in G}\{I(w)\}
$$

(c) if $F \subseteq \mathcal{X}$ is compact, open convex, closed convex, or any convex set in $\mathcal{B}(\mathcal{X})$ when $\mathcal{X}$ is finite-dimensional, then

$$
\limsup _{t \uparrow \infty} \frac{1}{t} \ln \mathbb{P}_{t}^{c}\left[\frac{W_{t}}{t} \in F\right] \leq-\inf _{w \in F}\{I(w)\} .
$$

Furthermore, if $\mathcal{X}$ is finite-dimensional, then this bound is valid for any closed set $F$ provided that $\mathbb{E}\left[e^{\xi\left\|X_{1}\right\|+v\left(S_{1}\right)-\zeta S_{1}} \mathbb{1}_{\left\{S_{1}<\infty\right\}}\right]<+\infty$ for some numbers $\zeta \geq 0$ and $\xi>0$.

The lower bound in part (b) and the upper bound in part (c) are called, respectively, large deviation lower bound and large deviation upper bound [12,13. When a lower semicontinuous function $I$ exists so that the large deviation lower bound holds for each open set $G$ and the large deviation upper bound holds for each compact set $F$, then $W_{t}$ is said to satisfy a weak large deviation principle (weak LDP) with rate function $I$ [12,13]. If the large deviation upper bound holds more generally for every closed set $F$, then $W_{t}$ is said to satisfy a full large deviation principle (full LDP) [12,13]. Theorem 1.1, states that the cumulative reward $W_{t}$ satisfies a weak LDP with rate function $I$ given by (1.2) within the CPM. If in addition $\mathcal{X}$ is finite-dimensional and the exponential moment condition $\mathbb{E}\left[e^{\xi\left\|X_{1}\right\|+v\left(S_{1}\right)-\zeta S_{1}} \mathbb{1}_{\left\{S_{1}<\infty\right\}}\right]<$ $+\infty$ is fulfilled for some $\zeta \geq 0$ and $\xi>0$, as certainly occurs in example 1.1 for any $\xi>0$ and $\zeta>M \xi+z_{o}$, then $W_{t}$ satisfies a full LDP. Regarding the validity of a full LDP for general infinite-dimensional Banach spaces $\mathcal{X}$, finding sufficient conditions is a harder problem that will be the focus of future studies. Trying to sketch an analogy with Cramér's theorem and the work by Donsker and Varadhan [6, one should probably investigate situations where there exists $\zeta \geq 0$ such that $\mathbb{E}\left[e^{\xi\left\|X_{1}\right\|+v\left(S_{1}\right)-\zeta S_{1}} \mathbb{1}_{\left\{S_{1}<\infty\right\}}\right]<+\infty$ for all $\xi>0$.

Let us move now to the PM, where there is no constraint on the last monomer. At variance with the CPM, the scaled cumulant generating function of $W_{t}$ may not exist in the PM, but the following proposition, which is proved in Section 3, shows that at least some bounds hold true. Set $\ell_{\mathrm{i}}:=\liminf \operatorname{i\uparrow }_{t \uparrow}(1 / t) \ln \mathbb{P}\left[S_{1}>t\right]$ and $\ell_{\mathrm{s}}:=\lim \sup _{t \uparrow \infty}(1 / t) \ln \mathbb{P}\left[S_{1}>\right.$ $t]$, and bear in mind that $-\infty \leq \ell_{\mathrm{i}} \leq \ell_{\mathrm{s}} \leq 0$.

Proposition 1.2. The following bounds hold for all $\varphi \in \mathcal{X}^{\star}$ :

$$
\begin{aligned}
z(\varphi) \vee \ell_{i} & \leq \liminf _{t \uparrow \infty} \frac{1}{t} \ln \mathbb{E}\left[e^{\varphi\left(W_{t}\right)+H_{t}}\right] \\
& \leq \limsup _{t \uparrow \infty} \frac{1}{t} \ln \mathbb{E}\left[e^{\varphi\left(W_{t}\right)+H_{t}}\right] \leq z(\varphi) \vee \ell_{s} .
\end{aligned}
$$


Denoting by $\mathbb{E}_{t}$ the expectation with respect to $\mathbb{P}_{t}$, proposition 1.2 entails that the limit $\lim _{t \uparrow \infty}(1 / t) \ln \mathbb{E}_{t}\left[e^{\varphi\left(W_{t}\right)}\right]$ exists, and equals $z(\varphi) \vee \ell_{\mathrm{s}}-z(0) \vee \ell_{\mathrm{S}}$, if either $\ell_{\mathrm{i}}=\ell_{\mathrm{S}}$ or $z(\varphi) \geq \ell_{\mathrm{s}}$. Thus, the scaled cumulant generating function of $W_{t}$ with respect to the PM is defined if either $\ell_{\mathrm{i}}=\ell_{\mathrm{s}}$, which includes the case $\ell_{\mathrm{s}}=-\infty$, or the condition $z(\varphi) \geq \ell_{\mathrm{s}}>-\infty$ is met for all $\varphi \in \mathcal{X}^{\star}$, as in the following example.

Example 1.2. The bound $z(\varphi) \geq \ell_{s}>-\infty$ holds for all $\varphi \in \mathcal{X}^{\star}$ if $\mathbb{P}\left[S_{1}<\infty\right]=1$, $\liminf _{s \uparrow \infty} v(s) / s=0$, and there exists a positive real function $g$ on $\{1,2, \ldots\} \cup\{\infty\}$ such that $\lim _{s \uparrow \infty} g(s) / s=0$ and $\left\|X_{1}\right\| \leq g\left(S_{1}\right)$ with full probability. Indeed, given any $\zeta<\ell_{s}$, under these hypotheses one can find $\epsilon>0$ such that $\zeta+\epsilon<\ell_{s} \leq 0$ and $-\|\varphi\| g(s)+v(s) \geq-\epsilon s$ for all sufficiently large $s$. Then, $\mathbb{E}\left[e^{\varphi\left(X_{1}\right)+v\left(S_{1}\right)-\zeta S_{1}} \mathbb{1}_{\left\{S_{1}<\infty\right\}}\right] \geq \sum_{s \geq 1} e^{-\|\varphi\| g(s)+v(s)-\zeta s} p(s) \geq$ $\sum_{s>t} e^{-(\zeta+\epsilon) s} p(s) \geq e^{-(\zeta+\epsilon) t} \mathbb{P}\left[S_{1}>t\right]$ for all sufficiently large $t$ as $\mathbb{P}\left[S_{1}=\infty\right]=0$. It follows that $\mathbb{E}\left[e^{\varphi\left(X_{1}\right)+v\left(S_{1}\right)-\zeta S_{1}} \mathbb{1}_{\left\{S_{1}<\infty\right\}}\right]=+\infty$ since $\zeta+\epsilon<\ell_{s}$, which results in $z(\varphi) \geq \ell_{s}$ according to definition (1.1).

In order to establish large deviation bounds with respect to the PM, it is convenient to distinguish the case $\ell_{\mathrm{s}}=-\infty$ from the case $\ell_{\mathrm{s}}>-\infty$. The following theorem, which represents our second main result, provides weak and full LDPs for the renewal-reward process $t \mapsto W_{t}$ with respect to the $\mathrm{PM}$ when $\ell_{\mathrm{s}}=-\infty$. The proof is given in Section 3 .

Theorem 1.2. Assume $\ell_{s}=-\infty$. The following conclusions hold:

(a) if $G \subseteq \mathcal{X}$ is open, then

$$
\liminf _{t \uparrow \infty} \frac{1}{t} \ln \mathbb{P}_{t}\left[\frac{W_{t}}{t} \in G\right] \geq-\inf _{w \in G}\{I(w)\}
$$

(b) if $F \subseteq \mathcal{X}$ is compact, then

$$
\limsup _{t \uparrow \infty} \frac{1}{t} \ln \mathbb{P}_{t}\left[\frac{W_{t}}{t} \in F\right] \leq-\inf _{w \in F}\{I(w)\} .
$$

If $F \subseteq \mathcal{X}$ is open convex, closed convex, or any convex set in $\mathcal{B}(\mathcal{X})$ when $\mathcal{X}$ is finitedimensional, then this bound is valid whenever $I(0)<+\infty$. Furthermore, if $\mathcal{X}$ is finitedimensional, then it is valid for any closed set $F$ provided that $\mathbb{E}\left[e^{\xi\left\|X_{1}\right\|+v\left(S_{1}\right)-\zeta S_{1}} \mathbb{1}_{\left\{S_{1}<\infty\right\}}\right]<$ $+\infty$ for some numbers $\zeta \geq 0$ and $\xi>0$.

In general, the large deviation upper bound in part (b) cannot be extended to convex sets if $\ell_{\mathrm{s}}=-\infty$ and $I(0)=+\infty$. Examples with an open convex set and a closed convex set where such bound fails will be shown at the end of Section 3 .

The case $\ell_{\mathrm{s}}>-\infty$ is more involved and calls for two rate functions, $I_{\mathrm{i}}$ and $I_{\mathrm{s}}$, which are defined for each $w \in \mathcal{X}$ by the formulas

$$
I_{\mathrm{i}}(w):=\sup _{\varphi \in \mathcal{X}^{\star}}\left\{\varphi(w)-z(\varphi) \vee \ell_{\mathrm{i}}+z(0) \vee \ell_{\mathrm{S}}\right\}
$$

and

$$
I_{\mathrm{S}}(w):=\sup _{\varphi \in \mathcal{X}^{\star}}\left\{\varphi(w)-z(\varphi) \vee \ell_{\mathrm{S}}+z(0) \vee \ell_{\mathrm{i}}\right\}
$$

The following theorem, which is our third and last main result, provides large deviation bounds with respect to the PM when $\ell_{\mathrm{s}}>-\infty$. The proof is reported in Section 3 .

Theorem 1.3. Assume $\ell_{s}>-\infty$. The following conclusions hold:

(a) the functions $I_{i}$ and $I_{s}$ are lower semicontinuous and proper convex;

(b) if $G \subseteq \mathcal{X}$ is open, then

$$
\liminf _{t \uparrow \infty} \frac{1}{t} \ln \mathbb{P}_{t}\left[\frac{W_{t}}{t} \in G\right] \geq-\inf _{w \in G}\left\{I_{i}(w)\right\} ;
$$


(c) if $F \subseteq \mathcal{X}$ is compact, open convex, closed convex, or any convex set in $\mathcal{B}(\mathcal{X})$ when $\mathcal{X}$ is finite-dimensional, then

$$
\limsup _{t \uparrow \infty} \frac{1}{t} \ln \mathbb{P}_{t}\left[\frac{W_{t}}{t} \in F\right] \leq-\inf _{w \in F}\left\{I_{s}(w)\right\} .
$$

Furthermore, if $\mathcal{X}$ is finite-dimensional, then this bound is valid for any closed set $F$ provided that $\mathbb{E}\left[e^{\xi\left\|X_{1}\right\|+v\left(S_{1}\right)-\zeta S_{1}} \mathbb{1}_{\left\{S_{1}<\infty\right\}}\right]<+\infty$ for some numbers $\zeta \geq 0$ and $\xi>0$.

Theorem 1.3 states that the renewal-reward process $t \mapsto W_{t}$ satisfies a weak LDP with rate function $I_{\mathrm{S}}$ within the $\mathrm{PM}$ provided that $I_{\mathrm{i}}=I_{\mathrm{s}}$. The exponential moment condition $\mathbb{E}\left[e^{\xi\left\|X_{1}\right\|+v\left(S_{1}\right)-\zeta S_{1}} \mathbb{1}_{\left\{S_{1}<\infty\right\}}\right]<+\infty$ for some $\zeta \geq 0$ and $\xi>0$ gives a full LDP with rate function $I_{\mathrm{s}}$ when $\mathcal{X}$ is finite-dimensional and $I_{\mathrm{i}}=I_{\mathrm{s}}$. We have $I_{\mathrm{i}}=I_{\mathrm{s}}$ if $\ell_{\mathrm{i}}=\ell_{\mathrm{s}}$, as expected in most applications, or if the condition $z(\varphi) \geq \ell_{\mathrm{s}}$ is fulfilled for all $\varphi \in \mathcal{X}^{\star}$, as in example 1.2. In the latter case, $I_{\mathrm{i}}=I_{\mathrm{s}}=I$.

\subsection{Discussion}

Large deviations for renewal-reward processes have been investigated by many authors over the past decades. Their attention has been focused on both discrete-time and continuoustime frameworks and, in most cases, on rewards taking real values. In order to fix ideas, when talking about renewal systems in the domain of time we think of a PM with waiting times satisfying $\mathbb{P}\left[S_{1}<\infty\right]=1$ and potential $v=0$. An almost omnipresent hypothesis in previous works is the Cramér condition $\mathbb{E}\left[e^{\xi\left\|X_{1}\right\|+\xi S_{1}}\right]<+\infty$ for some number $\xi>0$.

The simplest example of renewal-reward process has unit rewards and corresponds to the counting renewal process $t \mapsto N_{t}:=\sum_{i>1} \mathbb{1}_{\left\{T_{i} \leq t\right\}}$. Glynn and Whitt 14 investigated the connection between LDPs of the inverse processes $t \mapsto N_{t}$ and $i \mapsto T_{i}$, providing a full LDP for $N_{t}$ under the Cramér condition. This condition was later relaxed by Duffield and Whitt [18]. Jiang [20] studied the large deviations of the extended counting renewal process $t \mapsto \sum_{i>1} \mathbb{1}_{\left\{T_{i} \leq i^{\alpha} t\right\}}$ with $\alpha \in[0,1)$ under the Cramér condition. Glynn and Whitt [14] and Duffield and Whitt [18, together with Puhalskii and Whitt [17, also investigated the connection between sample-path LDPs of the processes $t \mapsto N_{t}$ and $i \mapsto T_{i}$ under the Cramér condition.

Starting from sample-path LDPs of inverse and compound processes, Duffy and RodgersLee [19] sketched a full LDP for renewal-reward processes with real rewards by means of the contraction principle under the stringent exponential moment condition $\mathbb{E}\left[e^{\xi\left\|X_{1}\right\|+\xi S_{1}}\right]<$ $+\infty$ for all $\xi>0$. Some full LDPs for real renewal-reward processes were later proposed by Macci [15, 16, under existence and essentially smoothness of the scaled cumulant generating function, which allow for an application of the Gärtner-Ellis theorem [12,13. Essentially smoothness of the scaled cumulant generating function has been recently relaxed by Borovkov and Mogulskii 21,22, which used the Cramér's theorem to establish a full LDP under the Cramér condition. Under the Cramér condition, they [23 25] have also obtained sample-path LDPs for real renewal-reward processes.

A different approach based on empirical measures has been considered by Lefevere, Mariani, and Zambotti [26], which have investigated large deviations for the empirical measures of forward and backward recurrence times associated with a renewal process, and have then derived by contraction a full LDP for renewal-rewards processes with rewards determined by the waiting times: $X_{i}:=f\left(S_{i}\right)$ for each $i$ with a bounded real function $f$. Later, Mariani and Zambotti [27] have developed a renewal version of Sanov's theorem by studying the empirical law of rewards that take values in a generic Polish space. By appealing to the contraction principle, this result could give a full LDP for a renewal-reward process with rewards valued in a real separable Banach space, but only provided that the strong exponential moment condition $\mathbb{E}\left[e^{\xi\left\|X_{1}\right\|}\right]<+\infty$ for all $\xi>0$ is satisfied as discussed by Schied [28.

We conclude this brief review of previous contributions by mentioning that a moderate deviation principle for real renewal-reward processes was obtained by Tsirelson [29] under an exponential moment condition. Exact asymptotics for the counting renewal process and real renewal-reward processes has been investigated under the Cramér condition and several 
additional smoothness hypotheses by Serfozo [30, Kuczek and Crank [31, Chi [32, and Borovkov and Mogulskii [33,34.

Previous works leave open the question of whether some large deviation principles free from exponential moment conditions can be established for renewal-reward processes, in the wake of the sharp version of Cramér's theorem demonstrated by Bahadur and Zabell $[5]$. The present paper gives a positive answer to this question at the price of restricting to the discrete-time framework. Indeed, through theorems 1.1, 1.2. and 1.3 we supply weak LDPs and large deviation upper bounds for measurable convex sets that are completely free from hypotheses. Moreover, when finite-dimensional rewards are considered, and when $\mathbb{P}\left[S_{1}<\infty\right]=1$ and $v=0$ to make a comparison with previous studies, we provide full LDPs under the exponential moment condition $\mathbb{E}\left[e^{\xi\left\|X_{1}\right\|-\zeta S_{1}}\right]<+\infty$ for some numbers $\zeta \geq 0$ and $\xi>0$, which is weaker than the Cramér condition $\mathbb{E}\left[e^{\xi\left\|X_{1}\right\|+\xi S_{1}}\right]<+\infty$ for some $\xi>0$. For instance, rewards of example 1.1 that define the macroscopic observables of Statistical Mechanics [11] always satisfy our weak exponential moment condition, whereas in general they do not fulfill the Cramér condition.

In order to drop exponential moment conditions, a novel approach with respect to past methods had to be devised to tackle the problem, and a new approach was suggested to us by the theory of polymer pinning [7,8]. This new approach is based on super-additivity, but requires discrete time to be implemented. It came from here the need to focus on the discretetime framework. In such framework, conditioning on the event that the last time is a renewal time is a meaningful procedure and enables a super-additivity property of renewal-reward processes to emerge. This procedure introduces a constrained model similarly to what is done with polymers. This way, we were able to find a successful strategy for investigating large deviations and we were naturally led to link renewal-reward processes to the PM and the CPM. Importantly, the CPM is not a merely mathematical tool to tackle the PM, but it also represents the renewal models of Statistical Mechanics [11, such as the PolandScheraga model, the Fisher-Felderhof model, the Wako-Saitô-Muñoz-Eaton model, and the Tokar-Dreyssé model. In this respect, the large deviation theory developed in this paper must be added to those already existing for other models of Statistical Mechanics, including the Curie-Weiss model [35, the Curie-Weiss-Potts model [36, the mean-field Blume-EmeryGriffiths model [37, and, to some extent, the Ising model as well as general Gibbs measures relative to an interaction potential [38, 41].

Going back for a moment to the domain of time with $\mathbb{P}\left[S_{1}<\infty\right]=1$ and $v=0$, it is interesting to point out that Duffy and Rodgers-Lee [19, Lefevere, Mariani, and Zambotti [26], and Borovkov and Mogulskii [21,22] found, with increasing level of generality, an apparently different rate function. They constructed the rate function for renewal-reward processes from the Cramér rate function $\Upsilon_{\mathrm{C}}$ of the waiting time and reward pair $\left(S_{1}, X_{1}\right)$, defined for each pair $(\beta, w) \in \mathbb{R} \times \mathcal{X}$ by

$$
\Upsilon_{\mathrm{C}}(\beta, w):=\sup _{(\zeta, \varphi) \in \mathbb{R} \times \mathcal{X}^{\star}}\left\{\varphi(w)-\beta \zeta-\ln \mathbb{E}\left[e^{\varphi\left(X_{1}\right)-\zeta S_{1}}\right]\right\} .
$$

Starting from $\Upsilon_{\mathrm{C}}$, they considered the function $\inf _{\gamma>0}\left\{\gamma \Upsilon_{\mathrm{C}}(\cdot / \gamma, \cdot / \gamma)\right\}$, whose lower-semicontinuous regularization $\Upsilon$ is given for every $(\beta, w) \in \mathbb{R} \times \mathcal{X}$ by

$$
\Upsilon(\beta, w):=\lim _{\delta \downarrow 0} \inf _{\alpha \in(\beta-\delta, \beta+\delta)} \inf _{u \in B_{w, \delta}} \inf _{\gamma>0}\left\{\gamma \Upsilon_{\mathrm{C}}(\alpha / \gamma, u / \gamma)\right\} .
$$

Here $B_{w, \delta}:=\{u \in \mathcal{X}:\|u-w\|<\delta\}$ is the open ball of center $w$ and radius $\delta$. Duffy and Rodgers-Lee [19] dealt with the case $\mathcal{X}=\mathbb{R}$ and $\ell_{\mathrm{s}}=-\infty$ under a strong exponential moment condition, obtaining the rate function $\Lambda:=\Upsilon(1, \cdot)$. In this case we have the rate function $I$ by theorem 1.2 with $z(\varphi)=\inf \left\{\zeta \in \mathbb{R}: \mathbb{E}\left[e^{\varphi\left(X_{1}\right)-\zeta S_{1}}\right] \leq 1\right\}$ for all $\varphi$ as $S_{1}<\infty$ with full probability and $v=0$. Lefevere, Mariani, and Zambotti [26] too found the rate function $\Lambda$ when $X_{1}=f\left(S_{1}\right)$ with a bounded real function $f$. This instance falls under the umbrella of example 1.2, and so we get again the rate function $I$ by theorem 1.3. Borovkov and Mogulskii [21,22 studied the case $\mathcal{X}=\mathbb{R}$ under the Cramér condition. They obtained the rate function $\Lambda$ when $\ell_{\mathrm{s}}=-\infty$ and the rate function $\Lambda_{\mathrm{s}}:=\inf _{\beta \in[0,1]}\left\{\Upsilon(\beta, \cdot)-(1-\beta) \ell_{\mathrm{s}}\right\}$ when $\ell_{\mathrm{i}}=\ell_{\mathrm{s}}>-\infty$ or $z(\varphi) \geq \ell_{\mathrm{s}}>-\infty$ for all $\varphi \in \mathcal{X}^{\star}$. In these cases we have the rate 
functions $I$ and $I_{\mathrm{s}}$, respectively, by theorem 1.2 and 1.3 . Despite different expressions, our results are consistent with all the findings of these authors. Indeed, while uniqueness of the rate function [12, 13] suggests that there is at least some situation where $I=\Lambda$ and $I_{\mathrm{s}}=\Lambda_{\mathrm{s}}$, a direct comparison shows that these identities hold in general, as established by the following lemma which is proved in $\mathrm{A}$.

Lemma 1.1. Assume that $\mathbb{P}\left[S_{1}<\infty\right]=1$ and that $v=0$. Then, the following conclusions hold for every $w \in \mathcal{X}$ :

(a) $I(w)=\Lambda(w):=\Upsilon(1, w) ;$

(b) $I_{s}(w)=\Lambda_{s}(w):=\inf _{\beta \in[0,1]}\left\{\Upsilon(\beta, w)-(1-\beta) \ell_{s}\right\}$ provided that $\ell_{s}>-\infty$.

As a final remark, we stress that Borovkov and Mogulskii 2122 opted for not introducing two different rate functions for the large deviation lower and upper bounds, thus considering only problems where $I_{\mathrm{i}}=I_{\mathrm{s}}$. At variance with them, we decided to provide optimal large deviation bounds with possibly different rate functions in order to even address situations where the tail of the waiting time distribution is very oscillating. For instance, a physical renewal model giving rise to two possibly different rate functions has been found by Lefevere, Mariani, and Zambotti [42,43] in the description of a free particle interacting with a heat bath.

\section{Proof of proposition 1.1 and theorem 1.1}

We prove proposition 1.1 and theorem 1.1 as follows. In Section 2.1 we show the existence of a weak LDP with a convex rate function. This is the step where convexity and superadditivity arguments come into play. In Section 2.2 we introduce the generalized renewal equation formalism, which allows us to express the scaled cumulative generating function in terms of the function $z$ defined by (1.1). Then, we use this formalism in Section 2.3 to also relate the rate function to $z$. Finally, in Section 2.4 we summarize the results linking them to proposition 1.1 and to parts (a), (b), and (c) of theorem 1.1 .

Our theory of large deviations take advantage of the fact that a renewal process forgets the past and starts over at every renewal. Concretely, this means that $\left(U_{\tau+t}, \Delta_{\tau}^{t} H, \Delta_{\tau}^{t} W\right)_{t \geq 1}$ with $\Delta_{\tau}^{t} H:=H_{\tau+t}-H_{\tau}$ and $\Delta_{\tau}^{t} W:=W_{\tau+t}-W_{\tau}$ is independent of $\left(H_{\tau}, W_{\tau}\right)$ and distributed as $\left(U_{t}, H_{t}, W_{t}\right)_{t \geq 1}$ conditional on the event that a given integer $\tau \geq 1$ is a renewal, namely

$$
\begin{aligned}
\mathbb{E}\left[\mathbb{1}_{\left\{\left(H_{\tau}, W_{\tau}\right) \in \cdot\right\}} \mathbb{1}_{\left\{\left(U_{\tau+t}, \Delta_{\tau}^{t} H, \Delta_{\tau}^{t} W\right)_{t \geq 1} \in \star\right\}} U_{\tau}\right]= & \mathbb{E}\left[\mathbb{1}_{\left\{\left(H_{\tau}, W_{\tau}\right) \in \cdot\right\}} U_{\tau}\right] \\
& \cdot \mathbb{E}\left[\mathbb{1}_{\left\{\left(U_{t}, H_{t}, W_{t}\right)_{t \geq 1} \in \star\right\}}\right] .
\end{aligned}
$$

A formal proof of (2.1) can be drawn by noticing that if $\tau=T_{n}$ for some positive integer $n$, then $T_{i} \leq \tau$ for each $i \leq n$ and $T_{i}>\tau$ for any $i>n$. It follows that $H_{\tau}=$ $\sum_{i=1}^{n} v\left(S_{i}\right)$ and $W_{\tau}=\sum_{i=1}^{n} X_{i}$, so that the random vector $\left(H_{\tau}, W_{\tau}\right)$ depends only on $\left(S_{1}, X_{1}\right), \ldots,\left(S_{n}, X_{n}\right)$. At the same time, for any $t \geq 1$ we have $U_{\tau+t}=\sum_{i \geq n+1} \mathbb{1}_{\left\{T_{i}=\tau+t\right\}}=$ $\sum_{i>1} \mathbb{1}_{\left\{S_{n+1}+\cdots+S_{n+i}=t\right\}}, \Delta_{\tau}^{t} H=\sum_{i \geq n+1} v\left(S_{i}\right) \mathbb{1}_{\left\{T_{i} \leq \tau+t\right\}}=\sum_{i>1} v\left(S_{n+i}\right) \mathbb{1}_{\left\{S_{n+1}+\cdots+S_{n+i} \leq t\right\}}$, and analogously $\Delta_{\tau}^{t} W=\sum_{i>n+1} X_{i} \mathbb{1}_{\left\{T_{i} \leq \tau+t\right\}}=\sum_{i>1} X_{n+i} \mathbb{1}_{\left\{S_{n+1}+\cdots+S_{n+i} \leq t\right\}}$, showing that the vector $\left(U_{\tau+t}, \Delta_{\tau}^{t} H, \Delta_{\tau}^{t} W\right)$ depends only on $\left(S_{n+1}, X_{n+1}\right),\left(S_{n+2}, X_{n+2}\right), \ldots$ through the same formula that connects $\left(U_{t}, H_{t}, W_{t}\right)$ to $\left(S_{1}, X_{1}\right),\left(S_{2}, X_{2}\right), \ldots$

\subsection{Weak LDP in the constrained setting}

We leave the normalizing constant $Z_{t}^{c}$ aside for the moment and focus on the measure $\mu_{t}$ over $\mathcal{B}(\mathcal{X})$ defined for each time $t \geq 1$ by

$$
\mu_{t}:=\mathbb{E}\left[\mathbb{1}_{\left\{\frac{W_{t}}{t} \in \cdot\right\}} U_{t} e^{H_{t}}\right] .
$$


We have $\mu_{t}(\mathcal{X})=\mathbb{E}\left[U_{t} e^{H_{t}}\right]=Z_{t}^{c}>0$ for all $t>t_{c}$ and some $t_{c} \geq 0$ thanks to assumption 1.1 about aperiodicity, as we have seen at the end of Section 1.1. Of fundamental importance is the following super-multiplicativity property, which is not fulfilled by $\mathbb{P}_{t}^{c}\left[W_{t} / t \in \cdot\right]=\mu_{t} / Z_{t}^{c}$ precisely because of normalization.

Lemma 2.1. Let $C \in \mathcal{B}(\mathcal{X})$ be convex and let $\tau \geq 1$ and $t \geq 1$ be two integers. Then, $\mu_{\tau+t}(C) \geq \mu_{\tau}(C) \cdot \mu_{t}(C)$.

Proof. Writing $W_{\tau+t} /(\tau+t)=\lambda W_{\tau} / \tau+(1-\lambda) \Delta_{\tau}^{t} W / t$ with $\lambda:=\tau /(\tau+t)$, we recognize that $W_{\tau+t} /(\tau+t) \in C$ whenever $W_{\tau} / \tau \in C$ and $\Delta_{\tau}^{t} W / t \in C$ since $C$ is convex. It follows that

$$
\begin{aligned}
& \mu_{\tau+t}(C)=\mathbb{E}\left[\mathbb{1}_{\left\{\frac{W_{\tau+t}}{\tau+t} \in C\right\}} U_{\tau+t} e^{H_{\tau+t}}\right] \\
& \geq \mathbb{E}\left[\mathbb{1}_{\left\{\frac{W_{\tau}}{\tau} \in C\right\}} \mathbb{1}_{\left\{\frac{\Delta_{\tau}^{t} W}{t} \in C\right\}} U_{\tau+t} e^{H_{\tau+t}}\right] \\
& =\mathbb{E}\left[\mathbb{1}_{\left\{\frac{W_{\tau}}{\tau} \in C\right\}} e^{H_{\tau}} \mathbb{1}_{\left\{\frac{\Delta_{\tau}^{t} W}{t} \in C\right\}} U_{\tau+t} e^{\Delta_{\tau}^{t} H}\right] \text {. }
\end{aligned}
$$

A looser lower bound is obtained by introducing the renewal indicator $U_{\tau}$ with the motivation that $\left(U_{\tau+t}, \Delta_{\tau}^{t} H, \Delta_{\tau}^{t} W\right)$ is independent of $\left(U_{\tau}, H_{\tau}, W_{\tau}\right)$ and distributed as $\left(U_{t}, H_{t}, W_{t}\right)$ when $\tau$ is a renewal. This way, invoking (2.1) we find

$$
\begin{aligned}
\mu_{\tau+t}(C) & \geq \mathbb{E}\left[\mathbb{1}_{\left.\left\{\frac{W_{\tau} \in C}{\tau} \in\right\}_{\tau} e^{H_{\tau}} \mathbb{1}_{\left\{\frac{\Delta_{\tau}^{t} W}{t} \in C\right\}} U_{\tau+t} e^{\Delta_{\tau}^{t} H}\right]}\right. \\
& =\mathbb{E}\left[\mathbb{1}_{\left\{\frac{W_{\tau}}{\tau} \in C\right\}} U_{\tau} e^{H_{\tau}}\right] \cdot \mathbb{E}\left[\mathbb{1}_{\left\{\frac{W_{t}}{t} \in C\right\}} U_{t} e^{H_{t}}\right] \\
& =\mu_{\tau}(C) \cdot \mu_{t}(C),
\end{aligned}
$$

which proves the lemma.

Super-multiplicativity, which becomes super-additivity once logarithms are taken, makes it possible to describe in general terms the exponential decay with $t$ of the measure $\mu_{t}$. To this purpose, we denote by $\mathcal{L}$ the extended real function over $\mathcal{B}(\mathcal{X})$ defined by the formula

$$
\mathcal{L}:=\sup _{t>t_{c}}\left\{\frac{1}{t} \ln \mu_{t}\right\}
$$

If $C \in \mathcal{B}(\mathcal{X})$ is convex, then the super-additivity of $\ln \mu_{t}(C)$ immediately gives $\lim \sup _{t \uparrow \infty}(1 / t) \ln \mu_{t}(C)=$ $\mathcal{L}(C)$. The following lemma improves this result when $C$ is open as well as convex. Hereafter we denote by $B_{w, \delta}:=\{u \in \mathcal{X}:\|u-w\|<\delta\}$ the open ball of center $w$ and radius $\delta$, which is an example of open convex set.

Lemma 2.2. Let $C \subseteq \mathcal{X}$ be open and convex. Then, $\lim _{t \uparrow \infty}(1 / t) \ln \mu_{t}(C)$ exists as an extended real number and is equal to $\mathcal{L}(C)$.

Proof. We shall show in a moment that the hypothesis that $C$ is open entails that either $\mu_{t}(C)=0$ for all $t>t_{c}$ or there exists $\tau \geq t_{c}$ such that $\mu_{t}(C)>0$ for all $t>\tau$. Lemma[2.2 is obvious in the first case. The second case is solved as follows. Pick an integer $s>t_{c}$. Then, fix an integer $\gamma \geq 1$ such that $\gamma s>\tau$ and a constant $M>-\infty$ such that $\ln \mu_{r}(C) \geq M$ when $\gamma s \leq r<2 \gamma s$, which exists because $\gamma s>\tau$. Expressing any $t \geq 2 \gamma s$ as $t=q \gamma s+r$ with $q \geq 1$ and $\gamma s \leq r<2 \gamma s$, super-additivity gives $\ln \mu_{t}(C) \geq q \gamma \ln \mu_{s}(C)+\ln \mu_{r}(C) \geq q \gamma \ln \mu_{s}(C)+$ $M$, thus showing that $\lim \inf _{t \uparrow \infty}(1 / t) \ln \mu_{t}(C) \geq(1 / s) \ln \mu_{s}(C)$. The arbitrariness of $s$ yields $\liminf \operatorname{in}_{t \infty}(1 / t) \ln \mu_{t}(C) \geq \sup _{s>t_{c}}\left\{(1 / s) \ln \mu_{s}(C)\right\}=: \mathcal{L}(C)$.

We now prove that either $\mu_{t}(C)=0$ for all $t>t_{c}$ or there exists $\tau \geq t_{c}$ with the property that $\mu_{t}(C)>0$ for all $t>\tau$. Assume that $\mu_{\tau_{o}}(C)>0$ for some $\tau_{o}>t_{c}$. To begin with, we notice that if for every $w \in C$ it were possible to find a number $\delta_{w}>0$ such that $\mu_{\tau_{o}}\left(B_{w, \delta_{w}}\right)=0$, then the open covering $\left\{B_{w, \delta_{w}}\right\}_{w \in C}$ of $C$ would contain a countable 
subcollection covering $C$ by separability of $\mathcal{X}$ and Lindelöf's lemma with the consequence that $\mu_{\tau_{o}}(C)=0$. This argument shows that there exists at least one point $w_{o} \in C$ such that $\mu_{\tau_{o}}\left(B_{w_{o}, \delta}\right)>0$ for all $\delta>0$. Since $C$ is open, there is $\delta_{o}>0$ such that $B_{w_{o}, 2 \delta_{o}} \subseteq C$. This way, we have constructed open balls $B_{k}:=B_{w_{o}, k \delta_{o}}$ so that $\mu_{\tau_{o}}\left(B_{1}\right)>0$ and $B_{2} \subseteq C$. Furthermore, since $\lim _{k \uparrow \infty} \mu_{r}\left(B_{k}\right)=\mu_{r}(\mathcal{X})=Z_{r}^{c}>0$ for all $r>t_{c}$, there exists an integer $k_{o} \geq 1$ such that $\mu_{r}\left(B_{k_{o}}\right)>0$ if $r$ satisfies $\tau_{o} \leq r<2 \tau_{o}$. Set $\tau:=2 k_{o} \tau_{o}$.

Let us pick an arbitrary $t>\tau$ and let us show that $\mu_{t}(C)>0$. The fact that $t>\tau \geq 2 \tau_{o}$ makes it possible to express $t$ as $t=q \tau_{o}+r$ with integers $q$ and $r$ such that $q \geq 1$ and $\tau_{o} \leq r<2 \tau_{o}$. We notice that $W_{t} / t \in B_{2}$ whenever $W_{q \tau_{o}} / q \tau_{o} \in B_{1}$ and $\Delta_{q \tau_{o}}^{r} W / r \in B_{k_{o}}$, as the following bounds demonstrate:

$$
\begin{aligned}
\left\|W_{t}-t w_{o}\right\| & \leq\left\|W_{q \tau_{o}}-q \tau_{o} w_{o}\right\|+\left\|\Delta_{q \tau_{o}}^{r} W-r w_{o}\right\| \\
& <\delta_{o}\left(q \tau_{o}+k_{o} r\right)<\delta_{o}\left(t+2 k_{o} \tau_{o}\right)=\delta_{o}(t+\tau)<2 \delta_{o} t .
\end{aligned}
$$

Then, recalling that $B_{2} \subseteq C$ we get

$$
\begin{aligned}
\mu_{t}(C) & \geq \mathbb{E}\left[\mathbb{1}_{\left\{\frac{W_{t}}{t} \in B_{2}\right\}} U_{t} e^{H_{t}}\right] \\
& \geq \mathbb{E}\left[\mathbb{1}_{\left\{\frac{W_{q \tau_{o}}}{q \tau_{o}} \in B_{1}\right\}} \mathbb{1}_{\left\{\frac{\Delta_{q \tau_{o}}^{r} W}{r} \in B_{k_{o}}\right\}} U_{t} e^{H_{t}}\right] \\
& =\mathbb{E}\left[\mathbb{1}_{\left\{\frac{\left.W_{q \tau_{o}} \in B_{1}\right\}}{q \tau_{o}}\right\}} e^{H_{q \tau_{o}}} \mathbb{1}_{\left\{\frac{\Delta_{q \tau_{o}}^{r} W}{r} \in B_{k_{o}}\right\}} U_{q \tau_{o}+r} e^{\Delta_{q \tau_{o}}^{r} H}\right] .
\end{aligned}
$$

As in the proof of lemma 2.2, a convenient looser lower bound is obtained by introducing $U_{q \tau_{o}}$. Since $\left(U_{q \tau_{o}+r}, \Delta_{q \tau_{o}}^{r} H, \Delta_{q \tau_{o}}^{r} W\right)$ is independent of $\left(U_{q \tau_{o}}, H_{q \tau_{o}}, W_{q \tau_{o}}\right)$ and distributed as $\left(U_{r}, H_{r}, W_{r}\right)$ when $q \tau_{o}$ is a renewal we find

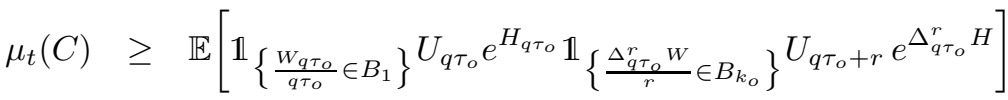

$$
\begin{aligned}
& =\mathbb{E}\left[\mathbb{1}_{\left\{\frac{\left.W_{q \tau_{o}} \in B_{1}\right\}}{q \tau_{o}}\right\}} U_{q \tau_{o}} e^{H_{q \tau_{o}}}\right] \cdot \mathbb{E}\left[\mathbb{1}_{\left\{\frac{W_{r}}{r} \in B_{k_{o}}\right\}} U_{r} e^{H_{r}}\right] \\
& =\mu_{q \tau_{o}}\left(B_{1}\right) \cdot \mu_{r}\left(B_{k_{o}}\right) \geq \mu_{\tau_{o}}^{q}\left(B_{1}\right) \cdot \mu_{r}\left(B_{k_{o}}\right) \text {, }
\end{aligned}
$$

where the last inequality is due to super-multiplicativity because $B_{1}$ is convex. We deduce from here that $\mu_{t}(C)>0$ as both $\mu_{\tau_{o}}\left(B_{1}\right)>0$ and $\mu_{r}\left(B_{k_{o}}\right)>0$ by construction.

Lemma 2.2 suggests to consider the putative rate function $J$ that maps any $w \in \mathcal{X}$ in the extended real number $J(w)$ defined by

$$
J(w):=-\inf _{\delta>0}\left\{\mathcal{L}\left(B_{w, \delta}\right)\right\} .
$$

In fact, the function $J$ controls the measure decay of open and compact sets as follows.

Proposition 2.1. The following conclusions hold:

(i) $\liminf _{t \uparrow \infty} \frac{1}{t} \ln \mu_{t}(G) \geq-\inf _{w \in G}\{J(w)\}$ for each $G \subseteq \mathcal{X}$ open;

(ii) $\limsup _{t \uparrow \infty} \frac{1}{t} \ln \mu_{t}(K) \leq-\inf _{w \in K}\{J(w)\}$ for each $K \subseteq \mathcal{X}$ compact.

Proof. Part (i) is immediate. Let $G \subseteq \mathcal{X}$ be open, let $w \in G$ be an arbitrary point, and let $\delta>0$ be such that $B_{w, \delta} \subseteq G$. Since $\mu_{t}(G) \geq \mu_{t}\left(B_{w, \delta}\right)$ and since $B_{w, \delta}$ is open and convex, lemma 2.2 gives $\liminf t \uparrow \infty(1 / t) \ln \mu_{t}(G) \geq \lim _{t \uparrow \infty}(1 / t) \ln \mu_{t}\left(B_{w, \delta}\right)=\mathcal{L}\left(B_{w, \delta}\right) \geq-J(w)$. The conclusion follows from the arbitrariness of $w$.

Moving to part (ii), pick a compact set $K$ in $\mathcal{X}$ and assume $\inf _{w \in K}\{J(w)\}>-\infty$, otherwise there is nothing to prove. Let $\lambda<\inf _{w \in K}\{J(w)\}$ be a real number. Since there exists $\epsilon>0$ such that $\lambda+\epsilon \leq J(w)=-\inf _{\delta>0}\left\{\mathcal{L}\left(B_{w, \delta}\right)\right\}$ for every $w \in K$, a number 
$\delta_{w}>0$ can be found for each $w \in K$ in such a way that $\mathcal{L}\left(B_{w, \delta_{w}}\right) \leq-\lambda$. Then, lemma 2.2 yields $\lim _{t \uparrow \infty}(1 / t) \ln \mu_{t}\left(B_{w, \delta_{w}}\right) \leq-\lambda$ for such $\delta_{w}$. Due to the compactness of $K$, there exist finitely many points $w_{1}, \ldots, w_{n}$ in $K$ such that $K \subseteq \cup_{i=1}^{n} B_{w_{i}, \delta_{w_{i}}}$. It follows that $\mu_{t}(K) \leq \sum_{i=1}^{n} \mu_{t}\left(B_{w_{i}, \delta_{w_{i}}}\right)$, which in turn gives $\lim \sup _{t \uparrow \infty}(1 / t) \ln \mu_{t}(K) \leq-\lambda$. This way, we get the desired upper bound by sending $\lambda$ to $\inf _{w \in K}\{J(w)\}$.

The first important properties of $J$ are presented in the following lemma.

Lemma 2.3. The function $J$ is lower semicontinuous and convex.

Proof. Pick $w \in \mathcal{X}$ and let $\left\{w_{i}\right\}_{i \geq 0}$ be a sequence of points converging to $w$. We show that $\liminf _{i \uparrow \infty} J\left(w_{i}\right) \geq-\mathcal{L}\left(B_{w, \delta}\right)$ for all numbers $\delta>0$, which results in $\liminf _{i \uparrow \infty} J\left(w_{i}\right) \geq J(w)$ and proves the lower semicontinuity of $J$. Given $\delta>0$ there exists $i_{o} \geq 0$ such that $\left\|w_{i}-w\right\| \leq \delta / 2$ if $i \geq i_{o}$. Then, monotonicity of $\mathcal{L}$ inherited from the measures $\mu_{t}$ entails that $-J\left(w_{i}\right) \leq \mathcal{L}\left(B_{w_{i}, \delta / 2}\right) \leq \mathcal{L}\left(B_{w, \delta}\right)$ for each $i \geq i_{o}$ since $B_{w_{i}, \delta / 2} \subseteq B_{w, \delta}$. The bound $\liminf \operatorname{i\uparrow \infty }_{i \uparrow} J\left(w_{i}\right) \geq-\mathcal{L}\left(B_{w, \delta}\right)$ follows from here.

As far as the proof of the convexity of $J$ is concerned, lower semicontinuity combined with the fact that dyadic rationals in $[0,1]$ are dense in $[0,1]$ makes it sufficient to verify that for each $u$ and $w$ in $\mathcal{X}$

$$
J\left(\frac{u+w}{2}\right) \leq \frac{J(u)+J(w)}{2}
$$

To this aim, we notice that for each number $\delta>0$ and integer $t \geq 1$ the conditions $W_{t} / t \in$ $B_{u, \delta}$ and $\Delta_{t}^{t} W / t \in B_{w, \delta}$ imply $W_{2 t} /(2 t) \in B_{(u+w) / 2, \delta}$, as one can easily verify. It follows that

$$
\begin{aligned}
\mu_{2 t}\left(B_{\frac{u+w}{2}, \delta}\right) & =\mathbb{E}\left[\mathbb{1}_{\left\{\frac{W_{2 t}}{2 t} \in B_{\frac{u+w}{2}, \delta}\right\}} U_{2 t} e^{H_{2 t}}\right] \\
& \geq \mathbb{E}\left[\mathbb{1}_{\left\{\frac{W_{t}}{t} \in B_{u, \delta}\right\}} \mathbb{1}_{\left\{\frac{\Delta_{t}^{t} W}{t} \in B_{w, \delta}\right\}} U_{2 t} e^{H_{2 t}}\right] \\
& =\mathbb{E}\left[\mathbb{1}_{\left\{\frac{W_{t}}{t} \in B_{u, \delta}\right\}} e^{H_{t}} \mathbb{1}_{\left\{\frac{\Delta_{t}^{t} W}{t} \in B_{w, \delta}\right\}} U_{2 t} e^{\Delta_{t}^{t} H}\right] .
\end{aligned}
$$

Inserting $U_{t}$ and exploiting the fact that $\left(U_{2 t}, \Delta_{t}^{t} H, \Delta_{t}^{t} W\right)$ is independent of $\left(U_{t}, H_{t}, W_{t}\right)$ and distributed as $\left(U_{t}, H_{t}, W_{t}\right)$ when $t$ is a renewal we get

$$
\begin{aligned}
\mu_{2 t}\left(B_{\frac{u+w}{2}, \delta}\right) & \geq \mathbb{E}\left[\mathbb{1}_{\left\{\frac{W_{t}}{t} \in B_{u, \delta}\right\}} U_{t} e^{H_{t}} \mathbb{1}_{\left\{\frac{\Delta_{t}^{t} W}{t} \in B_{w, \delta}\right\}} U_{2 t} e^{\Delta_{t}^{t} H}\right] \\
& =\mathbb{E}\left[\mathbb{1}_{\left\{\frac{W_{t}}{t} \in B_{u, \delta}\right\}} U_{t} e^{H_{t}}\right] \cdot \mathbb{E}\left[\mathbb{1}_{\left\{\frac{W_{t}}{t} \in B_{w, \delta}\right\}} U_{t} e^{H_{t}}\right] \\
& =\mu_{t}\left(B_{u, \delta}\right) \cdot \mu_{t}\left(B_{w, \delta}\right) .
\end{aligned}
$$

This way, taking logarithms, dividing by $2 t$, and sending $t$ to infinity, we find $\mathcal{L}\left(B_{(u+w) / 2, \delta}\right) \geq$ $(1 / 2) \mathcal{L}\left(B_{u, \delta}\right)+(1 / 2) \mathcal{L}\left(B_{w, \delta}\right) \geq-(1 / 2) J(u)-(1 / 2) J(w)$ thanks to lemma 2.2 because open balls are open convex sets. Inequality (2.2) follows from here by the arbitrariness of $\delta$.

We conclude the section strengthening proposition 2.1 for convex sets. We know that $\lim \sup _{t \uparrow \infty}(1 / t) \ln \mu_{t}(C)=\mathcal{L}(C)$ for every $C \in \mathcal{B}(\mathcal{X})$ convex thanks to super-additivity. The following lemma draws a link between $\mathcal{L}(C)$ and $\inf _{w \in C}\{J(w)\}$.

Lemma 2.4. Let $C \subseteq \mathcal{X}$ be open convex, closed convex, or any convex set in $\mathcal{B}(\mathcal{X})$ when $\mathcal{X}$ is finite-dimensional. Then, $\mathcal{L}(C) \leq-\inf _{w \in C}\{J(w)\}$.

Proof. The lemma is trivial if $\mathcal{L}(C)=-\infty$. Assume $\mathcal{L}(C)>-\infty$ and pick $\epsilon>0$. Since $\lim \sup _{t \uparrow \infty}(1 / t) \ln \mu_{t}(C)=\mathcal{L}(C)$ there exists an integer $\tau \geq 1$ such that $\mathcal{L}(C) \leq$ $(1 / \tau) \ln \mu_{\tau}(C)+\epsilon$. Completeness and separability of $\mathcal{X}$ entail that $\mu_{\tau}$ is tight as it is bounded from above by $Z_{\tau}^{c}<+\infty$ (see 44, theorem 7.1.7). Consequently, a compact set $K_{o} \subseteq C$ can be found so that $\mu_{\tau}(C) \leq \mu_{\tau}\left(K_{o}\right)+[1-\exp (-\epsilon \tau)] \mu_{\tau}(C)$. Thus, $\mu_{\tau}(C) \leq \exp (\epsilon \tau) \mu_{\tau}\left(K_{o}\right)$ and $\mathcal{L}(C) \leq(1 / \tau) \ln \mu_{\tau}\left(K_{o}\right)+2 \epsilon$ follows. We shall show in a moment that there exists a compact 
convex set $K$ with the property that $K_{o} \subseteq K \subseteq C$. Then, using the fact that $K_{o} \subseteq K$ we reach the further bound $\mathcal{L}(C) \leq(1 / \tau) \ln \mu_{\tau}(K)+2 \epsilon \leq \mathcal{L}(K)+2 \epsilon$. At this point, we notice that on the one hand $\mathcal{L}(K)=\lim \sup _{t \uparrow \infty}(1 / t) \ln \mu_{t}(K)$ by super-additivity as $K$ is convex, and on the other hand $\lim \sup _{t \uparrow \infty}(1 / t) \ln \mu_{t}(K) \leq-\inf _{w \in K}\{J(w)\}$ by part (ii) of proposition 2.1 as $K$ is compact. Thus, $\mathcal{L}(C) \leq-\inf _{w \in K}\{J(w)\}+2 \epsilon \leq-\inf _{w \in C}\{J(w)\}+2 \epsilon$ because $K \subseteq C$ and the lemma follows from the arbitrariness of $\epsilon$.

Let us prove now that there exists a compact convex set $K$ with the property that $K_{o} \subseteq$ $K \subseteq C$. The hypothesis that the convex set $C$ is either open or closed when $\mathcal{X}$ if infinitedimensional comes into play here. Let $C_{o}$ be the convex hull of $K_{o}$ and let $K:=\operatorname{cl} C_{o}, \operatorname{cl} A$ denoting the closure of a set $A$. Clearly, $K_{o} \subseteq C_{o} \subseteq C$ and $C_{o} \subseteq K$. Since $K_{o}$ is compact, $C_{o}$ is convex and compact whenever $\mathcal{X}$ is finite-dimensional, whereas $K$ is convex and compact in any circumstance (see [45, theorem 3.20). We want to demonstrate that $K \subseteq C$. If $\mathcal{X}$ is finite-dimensional, then $K=C_{o}$ and we get the desired result from $C_{o} \subseteq C$. If $\mathcal{X}$ is infinitedimensional and $C$ is closed, then $K \subseteq C$ follows from $C_{o} \subseteq C$ by taking closures. The only nontrivial case is when $\mathcal{X}$ is infinite-dimensional and $C$ is open. Assume that $C$ is open from now on and for each $w \in C$ let $\delta_{w}>0$ be such that $B_{w, 2 \delta_{w}} \subseteq C$. As $K_{o}$ is compact, there exist finitely many points $w_{1}, \ldots, w_{n}$ in $K_{o}$ so that $K_{o} \subseteq \cup_{i=1}^{n} B_{w_{i}, \delta_{w_{i}}}$. Let $K^{\prime}$ be the convex hull of $\cup_{i=1}^{n}\left(\operatorname{cl} B_{w_{i}, \delta_{w_{i}}} \cap K\right)$. We have $K^{\prime} \subseteq C$ because $\cup_{i=1}^{n}\left(\operatorname{cl} B_{w_{i}, \delta_{w_{i}}} \cap K\right) \subseteq \cup_{i=1}^{n} \mathrm{cl} B_{w_{i}, \delta_{w_{i}}} \subseteq C$ thanks to the fact that $B_{w_{i}, 2 \delta_{w_{i}}} \subseteq C$ for every $i$ and because $C$ is convex. This way, $K \subseteq C$ is verified if we show that $K=K^{\prime}$. The inclusion $K^{\prime} \subseteq K$ is immediate since $\cup_{i=1}^{n}\left(\operatorname{cl} B_{w_{i}, \delta_{w_{i}}} \cap K\right) \subseteq K$ and $K$ is convex. In order to show the opposite inclusion $K \subseteq K^{\prime}$ we observe that the set $K^{\prime}$ is convex and compact since it is the convex hull of the union of the compact convex sets $\mathrm{cl} B_{w_{1}, \delta_{w_{1}}} \cap K, \ldots, \operatorname{cl} B_{w_{n}, \delta_{w_{n}}} \cap K$ (see [45, theorem 3.20). Then, we observe that $K_{o} \subseteq K^{\prime}$ as $\cup_{i=1}^{n}\left(\operatorname{cl} B_{w_{i}, \delta_{w_{i}}} \cap K\right)=\left(\cup_{i=1}^{n} \operatorname{cl} B_{w_{i}, \delta_{w_{i}}}\right) \cap K$ and both $\cup_{i=1}^{n} \operatorname{cl} B_{w_{i}, \delta_{w_{i}}}$ and $K$ contain $K_{o}$. This way, we first realize that $C_{o} \subseteq K^{\prime}$ since $C_{o}$ is the smallest convex set that contains $K_{o}$, and by taking closures we later deduce that $K \subseteq K^{\prime}$ as $K^{\prime}$ is closed.

\subsection{Expectations and generalized renewal equation}

Let $\left(S_{1}, V_{1}\right),\left(S_{2}, V_{2}\right), \ldots$ be a sequence of independent and identically distributed random vectors on $(\Omega, \mathcal{F}, \mathbb{P})$, the $V_{i}$ 's taking values in $[0,+\infty)$, and for each time $t \geq 1$ denote by $\Psi_{t}$ the expected value

$$
\Psi_{t}:=\mathbb{E}\left[U_{t} \prod_{i \geq 1}\left(\mathbb{1}_{\left\{T_{i}>t\right\}}+V_{i} \mathbb{1}_{\left\{T_{i} \leq t\right\}}\right)\right] .
$$

Here we determine the asymptotic exponential rate of growth of $\Psi_{t}$ with respect to $t$. The solution to this problem is a needed preliminary step to relate the rate function $J$ to the function $z$ defined by (1.1). The computation of $\Psi_{t}$ takes advantage of the generalized renewal equation

$$
\Psi_{t}=\sum_{s=1}^{t} a_{s} \Psi_{t-s}
$$

satisfied for each $t \geq 1$ with the initial condition $\Psi_{0}:=1$, where $a_{s}:=\mathbb{E}\left[V_{1} \mathbb{1}_{\left\{S_{1}=s\right\}}\right]$ is a non-negative extended real number. This equation is deduced conditioning on $S_{1}$ and then using the fact that the renewal process starts over at the renewal time $T_{1}$. We are only interested in the case where $a_{\sigma_{l}}>0$ for each $l, \sigma_{1}, \ldots, \sigma_{m}$ being the $m$ coprime integers introduced in Section 1.1 to make effective aperiodicity of the waiting time distribution.

The expected value $A(\zeta):=\mathbb{E}\left[V_{1} e^{-\zeta S_{1}} \mathbb{1}_{\left\{S_{1}<\infty\right\}}\right]=\sum_{s>1} a_{s} e^{-\zeta s}$ exists as an extended real number and defines a lower semicontinuous function $A$ that maps $\zeta \in \mathbb{R}$ in $A(\zeta)$. The number $\psi$ given by

$$
\psi:=\inf \{\zeta \in \mathbb{R}: A(\zeta) \leq 1\}
$$

where the infimum over the empty set is customarily interpreted as $+\infty$, exactly is the exponential rate of growth we are looking for as stated by the next proposition. The level set $\{\zeta \in \mathbb{R}: A(\zeta) \leq 1\}$ is bounded from below since $A(\zeta) \geq \sum_{l=1}^{m} a_{\sigma_{l}} e^{-\zeta \sigma_{l}}>1$ for all $\zeta$ sufficiently negative and closed due to lower semicontinuity. Consequently, $\psi>-\infty$ and 
$A(\psi) \leq 1$ if $\psi<+\infty$. It follows that $\Psi_{t} \leq e^{\psi t}$ for all $t \geq 1$, which is trivial if $\psi=+\infty$ and is easily verified by induction starting from (2.4) when $\psi<+\infty$.

Proposition 2.2. $\lim _{t \uparrow \infty}(1 / t) \ln \Psi_{t}$ exists as an extended real number and is equal to $\psi>$ $-\infty$. Moreover, the bound $\Psi_{t} \leq e^{\psi t}$ holds for all $t \geq 1$.

Proof. The bound $\Psi_{t} \leq e^{\psi t}$ for all $t \geq 1$ gives $\lim \sup _{t \uparrow \infty}(1 / t) \ln \Psi_{t} \leq \psi$. Let us show that

$$
\liminf _{t \uparrow \infty} \frac{1}{t} \ln \Psi_{t} \geq \psi
$$

We have $\Psi_{t} \geq \mathbb{E}\left[U_{t} \prod_{i>1}\left(\mathbb{1}_{\left\{T_{i}>t\right\}}+V_{i} \mathbb{1}_{\left\{T_{i} \leq t\right\}}\right) \prod_{i=1}^{n} \mathbb{1}_{\left\{S_{i}=s_{i}\right\}}\right]=\prod_{i=1}^{n} a_{s_{i}}$ if $t=\sum_{i=1}^{n} s_{i}$. This way, the same arguments used in Section [1.1 to deduce $Z_{t}^{c}>0$ for all $t>t_{c}$ yield $\Psi_{t}>0$ for all $t>t_{c}$ as $a_{\sigma_{l}}>0$ by hypothesis for each $l$. This property allows us to prove (2.6) as follows. Pick a real number $\zeta<\psi$ and notice that there exists an integer $\tau \geq 1$ so that $\sum_{s=1}^{\tau} a_{s} e^{-\zeta s} \geq 1$. On the contrary we would have $A(\zeta) \leq 1$, which contradicts the assumption that $\zeta<\psi$. Since $\Psi_{t}>0$ for all $t>t_{c}$, we can find a constant $M>-\infty$ such that $\ln \Psi_{t} \geq M+\zeta t$ for every $t$ satisfying $t_{c}<t \leq t_{c}+\tau$. As a matter of fact, this bound is valid for all $t>t_{c}$. Indeed, an argument by induction based on the generalized renewal equation (2.4) shows that if $t>t_{c}+\tau$ and $\ln \Psi_{t-s} \geq M+\zeta(t-s)$ for any positive $s \leq \tau$, then

$$
\Psi_{t}=\sum_{s=1}^{t} a_{s} \Psi_{t-s} \geq \sum_{s=1}^{\tau} a_{s} \Psi_{t-s} \geq e^{M+\zeta t} \sum_{s=1}^{\tau} a_{s} e^{-\zeta s} \geq e^{M+\zeta t} .
$$

It follows that $\liminf \operatorname{in}_{t \uparrow \infty}(1 / t) \ln \Psi_{t} \geq \zeta$, giving (2.6) once $\zeta$ is sent to $\psi$.

The first application of proposition 2.2 we consider is concerned with the function $z$ defined by (1.1). To this aim we pick a linear functional $\varphi \in \mathcal{X}^{\star}$ and we set $V_{i}:=e^{\varphi\left(X_{i}\right)+v\left(S_{i}\right)}$ for every $i$. In this case, we have $a_{\sigma_{l}}=\mathbb{E}\left[e^{\varphi\left(X_{1}\right)+v\left(S_{1}\right)} \mathbb{1}_{\left\{S_{1}=\sigma_{l}\right\}}\right]>0$ for each $l$ as $p\left(\sigma_{l}\right)>0$ and $\Psi_{t}=\mathbb{E}\left[U_{t} e^{\varphi\left(W_{t}\right)+H_{t}}\right]$ for all $t$ since

$$
\prod_{i \geq 1}\left[\mathbb{1}_{\left\{T_{i}>t\right\}}+e^{\varphi\left(X_{i}\right)+v\left(S_{i}\right)} \mathbb{1}_{\left\{T_{i} \leq t\right\}}\right]=e^{\sum_{i \geq 1}\left[\varphi\left(X_{i}\right)+v\left(s_{i}\right)\right] \mathbb{1}_{\left\{T_{i} \leq t\right\}}}=e^{\varphi\left(W_{t}\right)+H_{t}} .
$$

Moreover, a direct comparison with (1.1) shows that the number $\psi$ associated with the present $V_{1}$ by formula (2.5) is nothing but $z(\varphi)$. Consequently, proposition 2.2 gives $\lim _{t \uparrow \infty}(1 / t) \ln \mathbb{E}\left[U_{t} e^{\varphi\left(W_{t}\right)+H_{t}}\right]=z(\varphi)$ and $z(\varphi)>-\infty$. It follows from here thanks to the arbitrariness of $\varphi$ that $z$ is convex and that $z$ never attains $-\infty$, thus resulting in a proper convex function since $z$ is finite at least in 0 due to assumption 1.2 as we have seen at the beginning of Section 1.2. Proposition 2.2 also shows that $\mathbb{E}\left[U_{t} e^{\varphi\left(W_{t}\right)+H_{t}}\right] \leq e^{z(\varphi) t}$ for all $t \geq 1$. The function $z$ is lower semicontinuous because if $\left\{\varphi_{i}\right\}_{i \geq 0}$ is a sequence converging to $\varphi$ and $t$ is any positive integer, then the bound $e^{z\left(\varphi_{i}\right) t} \geq \mathbb{E}\left[U_{t} e^{\varphi_{i}\left(W_{t}\right)+H_{t}}\right]$ and Fatou's lemma give $\liminf _{i \uparrow \infty} z\left(\varphi_{i}\right) \geq \liminf _{i \uparrow \infty}(1 / t) \ln \mathbb{E}\left[U_{t} e^{\varphi_{i}\left(W_{t}\right)+H_{t}}\right] \geq(1 / t) \ln \mathbb{E}\left[U_{t} e^{\varphi\left(W_{t}\right)+H_{t}}\right]$, which results in $\liminf _{i \uparrow \infty} z\left(\varphi_{i}\right) \geq z(\varphi)$ when $t$ is sent to infinity. We have thus proved the following lemma.

Lemma 2.5. The function $z$ is proper convex and lower semicontinuous. Given any $\varphi \in \mathcal{X}^{\star}$, the bound $\mathbb{E}\left[U_{t} e^{\varphi\left(W_{t}\right)+H_{t}}\right] \leq e^{z(\varphi) t}$ is valid for all $t \geq 1$ and the limit $\lim _{t \uparrow \infty}(1 / t) \ln \mathbb{E}\left[U_{t} e^{\varphi\left(W_{t}\right)+H_{t}}\right]=$ $z(\varphi)$ holds.

\subsection{Connection with the function $z$}

In this section we prove that the rate function $J$ is the Fenchel-Legendre transform of $z$, namely that $J(w)=\sup _{\varphi \in \mathcal{X}}\{\varphi(w)-z(\varphi)\}$ for all $w \in \mathcal{X}$. Lemma 2.3 states that $J$ is convex and lower semicontinuous. Actually, $J$ is proper convex. Indeed, by combining lemma 2.2 with $C:=\mathcal{X}$ and lemma 2.5 with $\varphi:=0$ we get $\mathcal{L}(\mathcal{X})=\lim _{t \uparrow \infty}(1 / t) \ln \mu_{t}(\mathcal{X})=$ $\lim _{t \uparrow \infty}(1 / t) \ln \mathbb{E}\left[U_{t} e^{H_{t}}\right]=z(0)$. This way, part (i) of proposition 2.1 with $G:=\mathcal{X}$ gives $z(0) \geq-\inf _{w \in \mathcal{X}}\{J(w)\}$ and lemma 2.4 with $C:=\mathcal{X}$ yields $z(0) \leq-\inf _{w \in \mathcal{X}}\{J(w)\}$, with 
the consequence that $\inf _{w \in \mathcal{X}}\{J(w)\}=-z(0)$. As $z(0)$ is finite, this equality shows that $J$ is finite at some point and that it never attains $-\infty$. Proper convexity and lower semicontinuity allow us to express $J$ in terms of its convex conjugate $J^{\star}$ as follows (see [46], theorem 2.3.3):

$$
J(w)=\sup _{\varphi \in \mathcal{X}^{\star}}\left\{\varphi(w)-J^{\star}(\varphi)\right\}
$$

for every $w \in \mathcal{X}$ with $J^{\star}(\varphi):=\sup _{w \in \mathcal{X}}\{\varphi(w)-J(w)\}$ for all $\varphi \in \mathcal{X}^{\star}$. This way, in order to demonstrate that $J$ is the Fenchel-Legendre transform of $z$ it suffices to show that $J^{\star}=z$. Basically, this argument is the same argument used by Cerf and Petit [4] for a short proof of Cramér's theorem in $\mathbb{R}$.

Proving the bound $J^{\star}(\varphi) \leq z(\varphi)$ for all $\varphi \in \mathcal{X}^{\star}$ is not difficult. To do this, we fix $\varphi \in \mathcal{X}^{\star}$ and we observe that lemma 2.5 together with the fact that $\varphi\left(W_{t}-t w\right) \geq-\left\|W_{t}-t w\right\|\|\varphi\| \geq$ $-t \delta\|\varphi\|$ if $W_{t} / t \in B_{w, \delta}$ gives for every $t \geq 1, w \in \mathcal{X}$, and $\delta>0$

$$
\begin{aligned}
e^{z(\varphi) t} \geq \mathbb{E}\left[U_{t} e^{\varphi\left(W_{t}\right)+H_{t}}\right] & \geq \mathbb{E}\left[\mathbb{1}_{\left\{\frac{W_{t}}{t} \in B_{w, \delta}\right\}} U_{t} e^{\varphi\left(W_{t}\right)+H_{t}}\right] \\
& =e^{t \varphi(w)} \mathbb{E}\left[\mathbb{1}_{\left\{\frac{W_{t}}{t} \in B_{w, \delta}\right\}} U_{t} e^{\varphi\left(W_{t}-t w\right)+H_{t}}\right] \\
& \geq e^{t \varphi(w)-t \delta\|\varphi\|} \mathbb{E}\left[\mathbb{1}_{\left\{\frac{W_{t}}{t} \in B_{w, \delta}\right\}} U_{t} e^{H_{t}}\right] \\
& =e^{t \varphi(w)-t \delta\|\varphi\|} \mu_{t}\left(B_{w, \delta}\right) .
\end{aligned}
$$

Taking logarithms, dividing by $t$, and sending $t$ to infinity, we get from here $z(\varphi) \geq \varphi(w)+$ $\mathcal{L}\left(B_{w, \delta}\right)-\delta\|\varphi\| \geq \varphi(w)-J(w)+\delta\|\varphi\|$ thanks to lemma 2.2. Thus, sending $\delta$ to zero first and appealing to the arbitrariness of $w$ later we reach the bound $z(\varphi) \geq \sup _{w \in \mathcal{X}}\{\varphi(w)-J(w)\}=$ : $J^{\star}(\varphi)$. A more sophisticated use of proposition 2.2 leads to the opposite bound, and hence to equality as stated by the following proposition.

Proposition 2.3. The convex conjugate $J^{\star}$ of $J$ equals $z$.

Proof. Pick a linear functional $\varphi \in \mathcal{X}^{\star}$. As $z(\varphi) \geq J^{\star}(\varphi)$, in order to show that $z(\varphi)=J^{\star}(\varphi)$ we must prove that $z(\varphi) \leq J^{\star}(\varphi)$. Assume that $J^{\star}(\varphi)<+\infty$, otherwise there is nothing to prove. We are going to obtain the bound $z(\varphi) \leq J^{\star}(\varphi)$ in two steps. At first we verify that for each $K \subseteq \mathcal{X}$ compact

$$
\limsup _{t \uparrow \infty} \frac{1}{t} \ln \mathbb{E}\left[\mathbb{1}_{\left\{\frac{W_{t}}{t} \in K\right\}} U_{t} e^{\varphi\left(W_{t}\right)+H_{t}}\right] \leq J^{\star}(\varphi) .
$$

Then, we demonstrate that for each real number $\zeta<z(\varphi)$ there exists a compact convex set $K \subseteq \mathcal{X}$ with the property that

$$
\zeta<\limsup _{t \uparrow \infty} \frac{1}{t} \ln \mathbb{E}\left[\mathbb{1}_{\left\{\frac{W_{t}}{t} \in K\right\}} U_{t} e^{\varphi\left(W_{t}\right)+H_{t}}\right] .
$$

The proposition follows by combining (2.9) with (2.8) first and by sending $\zeta$ to $z(\varphi)$ later.

Let us prove (2.8) for a given compact set $K$ in $\mathcal{X}$. Let $\lambda>J^{\star}(\varphi)$ and $\rho>0$ be two real numbers. Since there exists $\epsilon>0$ such that $\varphi(w)+\inf _{\delta>0}\left\{\mathcal{L}\left(B_{w, \delta}\right)\right\}=\varphi(w)-J(w) \leq$ $J^{\star}(\varphi) \leq \lambda-\epsilon$ for all $w$, for each $w \in \mathcal{X}$ we can find $\delta_{w}>0$ in such a way that $\delta_{w}\|\varphi\|<\rho$ and $\mathcal{L}\left(B_{w, \delta_{w}}\right) \leq \lambda-\varphi(w)$. Lemma 2.2 gives $\lim _{t \uparrow \infty}(1 / t) \ln \mu_{t}\left(B_{w, \delta_{w}}\right) \leq \lambda-\varphi(w)$ for such $\delta_{w}$. Furthermore, we have $\varphi\left(W_{t}-t w\right) \leq\left\|W_{t}-t w\right\|\|\varphi\| \leq t \delta_{w}\|\varphi\|<t \rho$ if $W_{t} / t \in B_{w, \delta_{w}}$. From the compactness of $K$ there exist finitely many points $w_{1}, \ldots, w_{n}$ in $K$ so that $K \subseteq \cup_{i=1}^{n} B_{w_{i}, \delta_{w_{i}}}$. It follows that for all $t \geq 1$

$$
\begin{aligned}
\mathbb{E}\left[\mathbb{1}_{\left\{\frac{W_{t}}{t} \in K\right\}} U_{t} e^{\varphi\left(W_{t}\right)+H_{t}}\right] & \leq \sum_{i=1}^{n} \mathbb{E}\left[\mathbb{1}_{\left\{\frac{W_{t}}{t} \in B_{w_{i}, \delta w_{i}}\right\}} U_{t} e^{\varphi\left(W_{t}\right)+H_{t}}\right] \\
& =\sum_{i=1}^{n} e^{t \varphi\left(w_{i}\right)} \mathbb{E}\left[\mathbb{1}_{\left\{\frac{W_{t}}{t} \in B_{w_{i}, \delta w_{i}}\right\}} U_{t} e^{\varphi\left(W_{t}-t w_{i}\right)+H_{t}}\right] \\
& \leq \sum_{i=1}^{n} \mu_{t}\left(B_{w_{i}, \delta_{w_{i}}}\right) e^{t \varphi\left(w_{i}\right)+t \rho}
\end{aligned}
$$


By combining this bound with $\lim _{t \uparrow \infty}(1 / t) \ln \mu_{t}\left(B_{w_{i}, \delta_{w_{i}}}\right) \leq \lambda-\varphi\left(w_{i}\right)$ for each $i$ we find

$$
\limsup _{t \uparrow \infty} \frac{1}{t} \ln \mathbb{E}\left[\mathbb{1}_{\left\{\frac{W_{t}}{t} \in K\right\}} U_{t} e^{\varphi\left(W_{t}\right)+H_{t}}\right] \leq \lambda+\rho .
$$

This way, we reach (2.8) by sending $\lambda$ to $J^{\star}(\varphi)$ and $\rho$ to 0 .

We now verify (2.9). Pick a real number $\zeta<z(\varphi)$ and observe that necessarily $\mathbb{E}\left[e^{\varphi\left(X_{1}\right)+v\left(S_{1}\right)-\zeta S_{1}} \mathbb{1}_{\left\{S_{1}<\infty\right\}}\right]>$ 1 by definition of $z(\varphi)$. Recall that $\mathbb{E}\left[e^{\varphi\left(X_{1}\right)+v\left(S_{1}\right)} \mathbb{1}_{\left\{S_{1}=\sigma_{l}\right\}}\right]>0$ for all $l$ since the $m$ coprime integers $\sigma_{1}, \ldots, \sigma_{m}$ satisfy $p\left(\sigma_{l}\right)>0$ for every $l$. We shall show at the end that there exists a compact convex set $K \subseteq \mathcal{X}$ such that

$$
\mathbb{E}\left[e^{\varphi\left(X_{1}\right)+v\left(S_{1}\right)-\zeta S_{1}} \mathbb{1}_{\left\{X_{1} / S_{1} \in K\right\}} \mathbb{1}_{\left\{S_{1}<\infty\right\}}\right]>1
$$

and

$$
\mathbb{E}\left[e^{\varphi\left(X_{1}\right)+v\left(S_{1}\right)} \mathbb{1}_{\left\{X_{1} / S_{1} \in K\right\}} \mathbb{1}_{\left\{S_{1}=\sigma_{l}\right\}}\right]>0
$$

for each $l$. This way, setting $V_{i}:=e^{\varphi\left(X_{i}\right)+v\left(S_{i}\right)} \mathbb{1}_{\left\{X_{i} / S_{i} \in K\right\}}$ for all $i$ and introducing the number $\psi$ defined by

$$
\psi:=\inf \left\{\eta \in \mathbb{R}: \mathbb{E}\left[V_{1} e^{-\eta S_{1}} \mathbb{1}_{\left\{S_{1}<\infty\right\}}\right] \leq 1\right\},
$$

we have $\zeta<\psi$ from (2.10). At the same time, if $a_{s}:=\mathbb{E}\left[V_{1} \mathbb{1}_{\left\{S_{1}=s\right\}}\right]$ for all $s$, then (2.11) gives $a_{\sigma_{l}}>0$ for each $l$. Consequently, we can invoke proposition 2.2 with the present $V_{i}$ to get

$$
\begin{aligned}
\zeta<\psi & =\lim _{t \uparrow \infty} \frac{1}{t} \ln \mathbb{E}\left[U_{t} \prod_{i \geq 1}\left(\mathbb{1}_{\left\{T_{i}>t\right\}}+V_{i} \mathbb{1}_{\left\{T_{i} \leq t\right\}}\right)\right] \\
& =\lim _{t \uparrow \infty} \frac{1}{t} \ln \mathbb{E}\left[U_{t} e^{\varphi\left(W_{t}\right)+H_{t}} \prod_{i \geq 1}\left(\mathbb{1}_{\left\{T_{i}>t\right\}}+\mathbb{1}_{\left\{X_{i} / S_{i} \in K\right\}} \mathbb{1}_{\left\{T_{i} \leq t\right\}}\right)\right] .
\end{aligned}
$$

On the other hand, as $K$ is convex, the condition $X_{i} / S_{i} \in K$ for all $i$ such that $T_{i} \leq$ $t$ entails $W_{t} / t \in K$ when $t$ is a renewal. To understand this point, we write $W_{t} / t=$ $\sum_{i>1}\left(X_{i} / S_{i}\right)\left(S_{i} / t\right) \mathbb{1}_{\left\{T_{i} \leq t\right\}}$ and we notice that when there exists a positive integer $n$ such that $T_{n}=t$, then $\sum_{i \geq 1}\left(S_{i} / t\right) \mathbb{1}_{\left\{T_{i} \leq t\right\}}=\sum_{i=1}^{n}\left(S_{i} / t\right)=T_{n} / t=1$. It follows that

$$
\begin{aligned}
\zeta & <\lim _{t \uparrow \infty} \frac{1}{t} \ln \mathbb{E}\left[U_{t} e^{\varphi\left(W_{t}\right)+H_{t}} \prod_{i \geq 1}\left(\mathbb{1}_{\left\{T_{i}>t\right\}}+\mathbb{1}_{\left\{X_{i} / S_{i} \in K\right\}} \mathbb{1}_{\left\{T_{i} \leq t\right\}}\right)\right] \\
& =\lim _{t \uparrow \infty} \frac{1}{t} \ln \mathbb{E}\left[U_{t} e^{\varphi\left(W_{t}\right)+H_{t}} \mathbb{1}_{\left\{\frac{W_{t}}{t} \in K\right\}} \prod_{i \geq 1}\left(\mathbb{1}_{\left\{T_{i}>t\right\}}+\mathbb{1}_{\left\{X_{i} / S_{i} \in K\right\}} \mathbb{1}_{\left\{T_{i} \leq t\right\}}\right)\right] \\
& \leq \limsup _{t \uparrow \infty} \frac{1}{t} \ln \mathbb{E}\left[U_{t} e^{\varphi\left(W_{t}\right)+H_{t}} \mathbb{1}_{\left\{\frac{W_{t}}{t} \in K\right\}}\right],
\end{aligned}
$$

which proves (2.9).

To conclude the proof of the proposition, we must show the validity of (2.10) and (2.11) for some compact convex set $K$. To this aim, consider the finite measure $\pi_{R}:=$ $\mathbb{E}\left[e^{\varphi\left(X_{1}\right)+v\left(S_{1}\right)-\zeta S_{1}} \mathbb{1}_{\left\{X_{1} / S_{1} \in \cdot\right\}} \mathbb{1}_{\left\{\left\|X_{1}\right\| \leq R\right\}} \mathbb{1}_{\left\{S_{1} \leq R\right\}}\right]$ on $\mathcal{B}(\mathcal{X}), R$ being a positive real number. The fact that $\mathbb{E}\left[e^{\varphi\left(X_{1}\right)+v\left(S_{1}\right)-\zeta S_{1}} \mathbb{1}_{\left\{S_{1}<\infty\right\}}\right]>1$ implies that there exists a sufficiently large $R$ so that $\pi_{R}(\mathcal{X})>1$ and completeness and separability of $\mathcal{X}$ entail that $\pi_{R}$ is tight (see 44, theorem 7.1.7). It follows that there exists a compact set $K_{O}$ such that $\pi_{R}\left(K_{o}\right)>1$, which gives $\mathbb{E}\left[e^{\varphi\left(X_{1}\right)+v\left(S_{1}\right)-\zeta S_{1}} \mathbb{1}_{\left\{X_{1} / S_{1} \in K_{o}\right\}} \mathbb{1}_{\left\{S_{1}<\infty\right\}}\right] \geq \pi_{R}\left(K_{o}\right)>1$. Similar arguments with $\pi_{R}:=\mathbb{E}\left[e^{\varphi\left(X_{1}\right)+v\left(S_{1}\right)} \mathbb{1}_{\left\{X_{1} / S_{1} \in \cdot\right\}} \mathbb{1}_{\left\{\left\|X_{1}\right\| \leq R\right\}} \mathbb{1}_{\left\{S_{1}=\sigma_{l}\right\}}\right]$ in combination with $\mathbb{E}\left[e^{\varphi\left(X_{1}\right)+v\left(S_{1}\right)} \mathbb{1}_{\left\{S_{1}=\sigma_{l}\right\}}\right]>0$ yield $\mathbb{E}\left[e^{\varphi\left(X_{1}\right)+v\left(S_{1}\right)} \mathbb{1}_{\left\{X_{1} / S_{1} \in K_{l}\right\}} \mathbb{1}_{\left\{S_{1}=\sigma_{l}\right\}}\right]>0$ for some compact set $K_{l}$. Let $K$ be the closed convex hull of $K_{o} \cup K_{1} \cup \cdots \cup K_{m}$. The set $K$ is convex and compact (see [45, theorem 3.20) and satisfies (2.10) and (2.11) as $K_{o} \subseteq K$ and $K_{l} \subseteq K$ for each $l$. 


\subsection{Proposition 1.1 and theorem 1.1 point by point}

In this section we explicitly verify proposition 1.1 and theorem 1.1 point by point, but the former simply is part of lemma 2.5 and does not need other demonstrations. Lemma 2.3 states that $J$ is convex and lower semicontinuous. Moreover, we have seen that $J$ is proper convex at the beginning of the last section. As $J(w)=\sup _{\varphi \in \mathcal{X}}\{\varphi(w)-z(\varphi)\}$ for all $w \in \mathcal{X}$ thanks to (2.7) and proposition [2.3) the rate function $I$ defined by (1.2) equals $J+z(0)$ and inherits the lower semicontinuity and proper convexity of $J$. These facts prove part (a) of theorem 1.1 Part (b) of theorem 1.1 follows from part (i) of propositions 2.1 bearing in mind that $\ln \mathbb{P}_{t}\left[W_{t} / t \in \cdot\right]=\ln \mu_{t}-\ln Z_{t}^{c}$ for each $t>t_{c}$, that $\lim _{t \uparrow \infty}(1 / t) \ln Z_{t}^{c}=\lim _{t \uparrow \infty} \mathbb{E}\left[U_{t} e^{H_{t}}\right]=z(0)$ by lemma 2.5, and that $I=J+z(0)$. Similarly, part (c) of theorem 1.1 concerning compact sets is due to part (ii) of proposition 2.1. Part (c) regarding convex sets follows from the limit $\lim \sup _{t \uparrow \infty}(1 / t) \ln \mu_{t}(C)=\mathcal{L}(C)$ valid for any $C \in \mathcal{B}(\mathcal{X})$ convex and lemma 2.4. Finally, part (c) for closed sets in the finite-dimensional case is demonstrated by the following proposition. Let us observe that the exponential moment condition $\mathbb{E}\left[e^{\xi\left\|X_{1}\right\|+v\left(S_{1}\right)-\zeta S_{1}} \mathbb{1}_{\left\{S_{1}<\infty\right\}}\right]<+\infty$ for some numbers $\zeta \geq 0$ and $\xi>0$ implies $z(\varphi)<+\infty$ for all $\varphi \in \mathcal{X}^{\star}$ such that $\|\varphi\| \leq \xi$. Indeed, the validity of this condition with certain $\zeta \geq 0$ and $\xi>0$ entails that a number $h$ large enough can be found so that $e^{-h} \mathbb{E}\left[e^{\xi\left\|X_{1}\right\|+v\left(S_{1}\right)-\zeta S_{1}} \mathbb{1}_{\left\{S_{1}<\infty\right\}}\right] \leq 1$. It follows that $\mathbb{E}\left[e^{\varphi\left(X_{1}\right)+v\left(S_{1}\right)-(\zeta+h) S_{1}} \mathbb{1}_{\left\{S_{1}<\infty\right\}}\right] \leq e^{-h} \mathbb{E}\left[e^{\xi\left\|X_{1}\right\|+v\left(S_{1}\right)-\zeta S_{1}} \mathbb{1}_{\left\{S_{1}<\infty\right\}}\right] \leq 1$ if $\|\varphi\| \leq \xi$ as $S_{1} \geq 1$ with full probability, which gives $z(\varphi)<+\infty$ according to definition (1.1). It is easy to verify that if $\mathcal{X}$ is finite-dimensional, then the above exponential moment condition is tantamount to the existence of $\xi>0$ such that $z(\varphi)<+\infty$ for all $\varphi$ fulfilling $\|\varphi\| \leq \xi$.

Proposition 2.4. Assume that $\mathcal{X}$ has finite dimension and that there exist numbers $\zeta \geq 0$ and $\xi>0$ such that $\mathbb{E}\left[e^{\xi\left\|X_{1}\right\|+v\left(S_{1}\right)-\zeta S_{1}} \mathbb{1}_{\left\{S_{1}<\infty\right\}}\right]<+\infty$. Then, for each $F \subseteq \mathcal{X}$ closed

$$
\limsup _{t \uparrow \infty} \frac{1}{t} \ln \mu_{t}(F) \leq-\inf _{w \in F}\{J(w)\}
$$

Proof. Fix a closed set $F$ in $\mathcal{X}$ and observe that $\inf _{w \in F}\{J(w)\} \geq \inf _{w \in \mathcal{X}}\{J(w)\}=-z(0)>$ $-\infty$. Then, pick a real number $\lambda<\inf _{w \in F}\{J(w)\}$. Let $d$ be the dimension of $\mathcal{X}$, let $\left\{w_{1}, \ldots, w_{d}\right\}$ be a basis of $\mathcal{X}$, and let $\left\{\vartheta_{1}, \ldots, \vartheta_{d}\right\} \subset \mathcal{X}^{\star}$ be the dual basis: $\vartheta_{i}\left(w_{j}\right)$ equals 1 if $i=j$ and 0 otherwise for all $i$ and $j$. For $i$ ranging from 1 to $d$, set $\varphi_{i}:=\vartheta_{i} /\left\|\vartheta_{i}\right\|$ and $\varphi_{d+i}:=-\varphi_{i}$. Since $z(\varphi)<+\infty$ if $\|\varphi\| \leq \xi, \xi>0$ being the number associated with the hypothesized exponential moment condition, there exists a real number $\rho>0$ with the property that $z\left(\xi \varphi_{i}\right)-\xi \rho \leq-\lambda$ for each $i$. Denoting by $K$ the compact set $K:=\cap_{i=1}^{2 d}\left\{w \in \mathcal{X}: \varphi_{i}(w) \leq \rho\right\}$, we have $K^{c}=\cup_{i=1}^{2 d}\left\{w \in \mathcal{X}: \varphi_{i}(w)>\rho\right\}$. This way, by making use of the Chernoff bound first and of the bound $\mathbb{E}\left[U_{t} e^{\xi \varphi_{i}\left(W_{t}\right)+H_{t}}\right] \leq e^{z\left(\xi \varphi_{i}\right) t}$ due to lemma 2.5 later, we obtain

$$
\begin{aligned}
\mu_{t}\left(K^{c}\right) & \leq \sum_{i=1}^{2 d} \mu_{t}\left(\left\{w \in \mathcal{X}: \varphi_{i}(w)>\rho\right\}\right)=\sum_{i=1}^{2 d} \mathbb{E}\left[\mathbb{1}_{\left\{\varphi_{i}\left(W_{t}\right)>\rho t\right\}} U_{t} e^{H_{t}}\right] \\
& \leq \sum_{i=1}^{2 d} \mathbb{E}\left[U_{t} e^{\xi \varphi_{i}\left(W_{t}\right)-\xi \rho t+H_{t}}\right] \leq \sum_{i=1}^{d} e^{z\left(\xi \varphi_{i}\right) t-\xi \rho t} \leq 2 d e^{-\lambda t}
\end{aligned}
$$

giving $\mu_{t}(F)=\mu_{t}(F \cap K)+\mu_{t}\left(F \cap K^{c}\right) \leq \mu_{t}(F \cap K)+2 d e^{-\lambda t}$ for each $t$. On the other hand, part (ii) of proposition 2.1 with the compact set $F \cap K$ shows that $\lim \sup _{t \uparrow \infty}(1 / t) \ln \mu_{t}(F \cap$ $K) \leq-\inf _{w \in F \cap K}\{J(w)\} \leq-\inf _{w \in F}\{J(w)\} \leq-\lambda$. It follows that limsup $\sup _{t \uparrow \infty}(1 / t) \ln \mu_{t}(F) \leq$ $-\lambda$, which proves the proposition once $\lambda$ is sent to $\inf _{w \in F}\{J(w)\}$.

\section{Proof of proposition 1.2 and of theorems 1.2 and 1.3}

Large deviation bounds within the PM can be made a consequence of the corresponding bounds in the CPM by exploiting conditioning as follows. Pick an integer time $t \geq 1$ and notice that if $T_{1} \leq t$, then there is one and only one positive integer $n \leq t$ such that $T_{n} \leq t$ 
and $T_{n+1}>t$. Thus, $\Omega=\left\{T_{1}>t\right\} \cup\left\{T_{1} \leq t\right\}$ and $\left\{T_{1} \leq t\right\}=\cup_{n=1}^{t}\left\{T_{n} \leq t\right.$ and $\left.T_{n+1}>t\right\}=$ $\cup_{n=1}^{t} \cup_{\tau=n}^{t}\left\{T_{n}=\tau\right.$ and $\left.T_{n+1}>t\right\}$, the events $\left\{T_{n}=\tau\right.$ and $\left.T_{n+1}>t\right\}$ for $1 \leq n \leq \tau \leq t$ being disjoint. The condition $T_{1}>t$ is tantamount to $S_{1}>t$ and implies that $H_{t}=0$ and $W_{t}=0$. The condition $T_{n}=\tau$ and $T_{n+1}>t$ is tantamount to $T_{n}=\tau$ and $S_{n+1}>t-\tau$ and implies that $H_{t}=\sum_{i=1}^{n} v\left(S_{i}\right)=H_{\tau}$ and $W_{t}=\sum_{i=1}^{n} X_{i}=W_{\tau}$ are independent of $S_{n+1}$. This way, for every $\varphi \in \mathcal{X}^{\star}$ we find the identity between measures

$$
\begin{aligned}
\mathbb{E}\left[\mathbb{1}_{\left\{W_{t} \in \cdot\right\}} e^{\varphi\left(W_{t}\right)+H_{t}}\right] & =\mathbb{E}\left[\mathbb{1}_{\left\{W_{t} \in \cdot\right\}} \mathbb{1}_{\left\{S_{1}>t\right\}} e^{\varphi\left(W_{t}\right)+H_{t}}\right] \\
& +\sum_{n=1}^{t} \sum_{\tau=n}^{t} \mathbb{E}\left[\mathbb{1}_{\left\{W_{t} \in \cdot\right\}} \mathbb{1}_{\left\{T_{n}=\tau\right\}} \mathbb{1}_{\left\{S_{n+1}>t-\tau\right\}} e^{\varphi\left(W_{t}\right)+H_{t}}\right] \\
& =\mathbb{1}_{\{0 \in \cdot\}} \cdot \mathbb{P}\left[S_{1}>t\right] \\
& +\sum_{\tau=1}^{t} \sum_{n=1}^{\tau} \mathbb{E}\left[\mathbb{1}_{\left\{W_{\tau} \in \cdot\right\}} \mathbb{1}_{\left\{T_{n}=\tau\right\}} e^{\varphi\left(W_{\tau}\right)+H_{\tau}}\right] \cdot \mathbb{P}\left[S_{1}>t-\tau\right] \\
& =\mathbb{1}_{\{0 \in \cdot\}} \cdot \mathbb{P}\left[S_{1}>t\right] \\
& +\sum_{\tau=1}^{t} \mathbb{E}\left[\mathbb{1}_{\left\{W_{\tau} \in \cdot\right\}} U_{\tau} e^{\varphi\left(W_{\tau}\right)+H_{\tau}}\right] \cdot \mathbb{P}\left[S_{1}>t-\tau\right]
\end{aligned}
$$

Formula (3.1) connects the free setting with the constrained setting and is the starting point to prove proposition 1.2 and theorems 1.2 and 1.3 . Once again, we leave normalization aside at the beginning and focus on the measure $\nu_{t}:=Z_{t} \mathbb{P}_{t}\left[W_{t} / t \in \cdot\right]$ on $\mathcal{B}(\mathcal{X})$. Identity (3.1) with $\varphi=0$ results in the expression

$$
\begin{aligned}
\nu_{t} & =\mathbb{E}\left[\mathbb{1}_{\left\{\frac{W_{t}}{t} \in \cdot\right\}} e^{H_{t}}\right] \\
& =\mathbb{1}_{\{0 \in \cdot\}} \cdot \mathbb{P}\left[S_{1}>t\right]+\sum_{\tau=1}^{t} \mathbb{E}\left[\mathbb{1}_{\left\{\frac{W_{\tau}}{t} \in \cdot\right\}} U_{\tau} e^{H_{\tau}}\right] \cdot \mathbb{P}\left[S_{1}>t-\tau\right] .
\end{aligned}
$$

We use this expression to derive a lower large deviation bound in Section 3.1 and an upper large deviation bound in Section 3.2. Theorem 1.2 is verified point by point in Section 3.3. where two counterexamples are also shown to demonstrate that the upper large deviation bound for open convex sets and closed convex sets cannot hold in general when $\ell_{\mathrm{s}}=-\infty$ and $I(0)=+\infty$. Finally, theorem 1.3 is verified point by point in Section 3.4 .

Regarding proposition 1.2, it is an immediate consequence of formula (3.1), which entails $\mathbb{E}\left[e^{\varphi\left(W_{t}\right)+H_{t}}\right]=\mathbb{P}\left[S_{1}>t\right]+\sum_{\tau=1}^{t} \mathbb{E}\left[U_{\tau} e^{\varphi\left(W_{\tau}\right)+H_{\tau}}\right] \cdot \mathbb{P}\left[S_{1}>t-\tau\right]$ for each $t$ and $\varphi$. Indeed, recalling the definitions $\lim \inf _{t \uparrow \infty}(1 / t) \ln \mathbb{P}\left[S_{1}>t\right]=: \ell_{\mathrm{i}}$ and $\lim \sup _{t \uparrow \infty}(1 / t) \ln \mathbb{P}\left[S_{1}>t\right]=$ : $\ell_{\mathrm{s}}$, as well as the $\operatorname{limit}_{\lim _{t \uparrow \infty}}(1 / t) \ln \mathbb{E}\left[U_{t} e^{\varphi\left(W_{t}\right)+H_{t}}\right]=z(\varphi)$ due to lemma 2.5. for all $\varphi \in \mathcal{X}^{\star}$ we get

$$
\liminf _{t \uparrow \infty} \frac{1}{t} \ln \mathbb{E}\left[e^{\varphi\left(W_{t}\right)+H_{t}}\right] \geq z(\varphi) \vee \ell_{\mathrm{i}}
$$

and

$$
\limsup _{t \uparrow \infty} \frac{1}{t} \ln \mathbb{E}\left[e^{\varphi\left(W_{t}\right)+H_{t}}\right] \leq z(\varphi) \vee \ell_{\mathrm{s}}
$$

\subsection{The lower large deviation bound}

In this section we prove the following lower bound without restrictions on $\ell_{\mathrm{i}}$ and $\ell_{\mathrm{s}}$.

Proposition 3.1. For each $G \subseteq \mathcal{X}$ open

$$
\liminf _{t \uparrow \infty} \frac{1}{t} \ln \nu_{t}(G) \geq-\inf _{w \in G} \sup _{\varphi \in \mathcal{X}^{\star}}\left\{\varphi(w)-z(\varphi) \vee \ell_{i}\right\} .
$$


Proof. Pick an open set $G$ in $\mathcal{X}$. In order to demonstrate the proposition it suffices to verify that for all $w \in G$

$$
\liminf _{t \uparrow \infty} \frac{1}{t} \ln \nu_{t}(G) \geq-\sup _{\varphi \in \mathcal{X}^{\star}}\left\{\varphi(w)-z(\varphi) \vee \ell_{\mathrm{i}}\right\} .
$$

This bound is immediate when $\ell_{\mathrm{i}}=-\infty$. Indeed, keeping only the term corresponding to $\tau=t$ in the r.h.s. of (3.2) we get $\nu_{t}(G) \geq \mu_{t}(G)$, which shows that $\lim \inf _{t \uparrow \infty}(1 / t) \ln \nu_{t}(G) \geq$ $-J(w)$ for any $w \in G$ thanks to part (i) of proposition 2.1. On the other hand, $J(w)$ is the r.h.s. of (3.5) if $\ell_{\mathrm{i}}=-\infty$ by formula (2.7) and proposition 2.3.

The proof of (3.5) is more laborious when $\ell_{\mathrm{i}}>-\infty$ and we assume that $\ell_{\mathrm{i}}>-\infty$ from now on. Let $\operatorname{dom} z:=\left\{\varphi \in \mathcal{X}^{\star}: z(\varphi)<+\infty\right\}$ be the effective domain of $z$ and consider the function $F$ that for a given $w \in G$ maps $(\beta, \varphi) \in[0,1] \times \operatorname{dom} z$ in the real number $F(\beta, \varphi):=$ $\varphi(w)-\beta z(\varphi)-(1-\beta) \ell_{\mathrm{i}}$. The function $F$ is concave and upper semicontinuous with respect to $\varphi$ for each fixed $\beta \in[0,1]$, inheriting these properties from $z$, and convex and continuous with respect to $\beta$ for each fixed $\varphi \in \operatorname{dom} z$. Then, due to compactness of the closed interval $[0,1]$, Sion's minimax theorem allows us to exchange the infimum over $\beta \in[0,1]$ and the supremum over $\varphi \in \operatorname{dom} z: \sup _{\varphi \in \operatorname{dom} z} \inf _{\beta \in[0,1]}\{F(\beta, \varphi)\}=\inf _{\beta \in[0,1]} \sup _{\varphi \in \operatorname{dom} z}\{F(\beta, \varphi)\}$. As $\inf _{\beta \in[0,1]}\left\{\varphi(w)-\beta z(\varphi)-(1-\beta) \ell_{\mathrm{i}}\right\}=\varphi(w)-z(\varphi) \vee \ell_{\mathrm{i}}$ and $z(\varphi) \vee \ell_{\mathrm{i}}=+\infty$ when $\varphi \notin \operatorname{dom} z$, this identity can be written as

$$
\sup _{\varphi \in \mathcal{X}^{\star}}\left\{\varphi(w)-z(\varphi) \vee \ell_{\mathrm{i}}\right\}=\inf _{\beta \in[0,1]} \sup _{\varphi \in \operatorname{dom} z}\left\{\varphi(w)-\beta z(\varphi)-(1-\beta) \ell_{\mathrm{i}}\right\} .
$$

This way, we get the bound (3.5) if we prove that for every $w \in G$ and $\beta \in[0,1]$

$$
\liminf _{t \uparrow \infty} \frac{1}{t} \ln \nu_{t}(G) \geq-\sup _{\varphi \in \operatorname{dom} z}\left\{\varphi(w)-\beta z(\varphi)-(1-\beta) \ell_{\mathrm{i}}\right\} .
$$

We prove (3.6) considering the case $\beta>0$ first. Pick a point $w \in G$ and a number $\beta \in(0,1]$ and denote by $\tau_{t}$ the greatest integer that is less than or equal to $\beta t$. Let $\delta>0$ be such that $B_{w, 2 \delta} \subseteq G$ and focus on all those sufficiently large integers $t$ such that $\tau_{t}>0$ and $\|w\|<\beta \delta t$. Within this setting, we have that the event $W_{\tau_{t}} / \tau_{t} \in B_{w / \beta, \delta}$ implies $W_{\tau_{t}} / t \in B_{w, 2 \delta} \subseteq G$. Indeed, since $0 \leq t-\tau_{t} / \beta<1 / \beta$ and $\|w\|<\beta \delta t$ we find $\left\|W_{\tau_{t}}-t w\right\| \leq\left\|W_{\tau_{t}}-\left(\tau_{t} / \beta\right) w\right\|+\left(t-\tau_{t} / \beta\right)\|w\|<\left\|W_{\tau_{t}}-\left(\tau_{t} / \beta\right) w\right\|+\delta t$. It follows that if $\left\|W_{\tau_{t}}-\left(\tau_{t} / \beta\right) w\right\|<\delta \tau_{t}$, then $\left\|W_{\tau_{t}}-t w\right\|<\delta \tau_{t}+\delta t \leq 2 \delta t$. This way, keeping only the term corresponding to $\tau=\tau_{t}>0$ in the r.h.s. of (3.2), we obtain

$$
\begin{aligned}
\nu_{t}(G) & \geq \mathbb{E}\left[\mathbb{1}_{\left\{\frac{W_{\tau_{t}}}{t} \in G\right\}} U_{\tau_{t}} e^{H_{t}}\right] \cdot \mathbb{P}\left[S_{1}>t-\tau_{t}\right] \\
& \geq \mathbb{E}\left[\mathbb{1}_{\left\{\frac{W_{\tau_{t}}}{\tau_{t}} \in B_{w / \beta, \delta}\right\}} U_{\tau_{t}} e^{H_{t}}\right] \cdot \mathbb{P}\left[S_{1}>t-\tau_{t}\right] \\
& =\mu_{\tau_{t}}\left(B_{\frac{w}{\beta}, \delta}\right) \cdot \mathbb{P}\left[S_{1}>t-\tau_{t}\right] .
\end{aligned}
$$

We have $\lim _{t \uparrow \infty}\left(1 / \tau_{t}\right) \ln \mu_{\tau_{t}}\left(B_{w / \beta, \delta}\right)=\mathcal{L}\left(B_{w / \beta, \delta}\right) \geq-J(w / \beta)$ by lemma 2.2 We also have $\lim _{t \uparrow \infty} \tau_{t} / t=\beta$ and $\liminf _{t \uparrow \infty}(1 / t) \ln \mathbb{P}\left[S_{1}>t-\tau_{t}\right]=(1-\beta) \ell_{\mathrm{i}}$. The latter limit is trivial in the case $\beta=1$ to which $\tau_{t}=t$ corresponds, whereas it follows from $\lim \inf _{t \uparrow \infty}(1 / t) \ln \mathbb{P}\left[S_{1}>\right.$ $t]=: \ell_{\mathrm{i}}$ when $\beta<1$ due to the fact that $t-\tau_{t}$ is now diverging as $t$ is sent to infinity. These arguments in combination with (3.7) prove that

$$
\begin{aligned}
\liminf _{t \uparrow \infty} \frac{1}{t} \ln \nu_{t}(G) & \geq-\beta J(w / \beta)+(1-\beta) \ell_{\mathrm{i}} \\
& =-\sup _{\varphi \in \mathcal{X}^{\star}}\{\varphi(w)-\beta z(\varphi)\}+(1-\beta) \ell_{\mathrm{i}} \\
& =-\sup _{\varphi \in \operatorname{dom} z}\left\{\varphi(w)-\beta z(\varphi)-(1-\beta) \ell_{\mathrm{i}}\right\},
\end{aligned}
$$

which is (3.6) under the hypothesis that $\beta>0$. 
In order to settle the case $\beta=0$, we take a point $u \in \mathcal{X}$ such that $c:=J(u)$ is finite, which exists because $J$ is proper convex. We have $z(\varphi)=J^{\star}(\varphi):=\sup _{w \in \mathcal{X}}\{\varphi(w)-J(w)\} \geq$ $\varphi(u)-c$ for all $\varphi \in \mathcal{X}^{\star}$ by proposition 2.3. As $G$ is open, for a given $w \in G$ we can find a number $\delta \in(0,1)$ such that $w+\epsilon u \in G$ whenever $\epsilon \in(0, \delta)$. Then, the bound (3.6) applies with a positive $\epsilon<\delta<1$ in place of $\beta$ and $w+\epsilon u$ in place of $w$ to give

$$
\begin{aligned}
\liminf _{t \uparrow \infty} \frac{1}{t} \ln \nu_{t}(G) & \geq-\sup _{\varphi \in \operatorname{dom} z}\left\{\varphi(w+\epsilon u)-\epsilon z(\varphi)-(1-\epsilon) \ell_{\mathrm{i}}\right\} \\
& \geq-\sup _{\varphi \in \operatorname{dom} z}\left\{\varphi(w)-\ell_{\mathrm{i}}\right\}-\epsilon\left(c+\ell_{\mathrm{i}}\right) .
\end{aligned}
$$

We obtain (3.6) corresponding to $\beta=0$ from here by sending $\epsilon$ to zero.

\subsection{The upper large deviation bound}

An upper large deviation bound for compact sets can be proved by means of standard arguments from large deviation theory without distinguishing the case $\ell_{\mathrm{s}}>-\infty$ from the case $\ell_{\mathrm{s}}=-\infty$. The following result holds.

Proposition 3.2. For each compact set $K \subseteq \mathcal{X}$

$$
\limsup _{t \uparrow \infty} \frac{1}{t} \ln \nu_{t}(K) \leq-\inf _{w \in K} \sup _{\varphi \in \mathcal{X}^{\star}}\left\{\varphi(w)-z(\varphi) \vee \ell_{s}\right\} .
$$

Proof. Let $K$ be a compact set in $\mathcal{X}$ and notice that $\inf _{w \in K} \sup _{\varphi \in \mathcal{X}}\left\{\varphi(w)-z(\varphi) \vee \ell_{\mathrm{s}}\right\} \geq$ $-z(0) \vee \ell_{\mathrm{s}}>-\infty$. Let $\lambda<\inf _{w \in K} \sup _{\varphi \in \mathcal{X}^{*}}\left\{\varphi(w)-z(\varphi) \vee \ell_{\mathrm{s}}\right\}$ and $\rho>0$ be real numbers. As there exists $\epsilon>0$ such that $\sup _{\varphi \in \mathcal{X}^{\star}}\left\{\varphi(w)-z(\varphi) \vee \ell_{\mathrm{s}}\right\} \geq \lambda+\epsilon$ for all $w \in K$, a linear functional $\varphi_{w} \in \mathcal{X}^{\star}$ can be found for each $w \in K$ with the property that $\varphi_{w}(w)-z\left(\varphi_{w}\right) \vee \ell_{\mathrm{s}} \geq$ $\lambda$. It is manifest that $z\left(\varphi_{w}\right)<+\infty$ for such $\varphi_{w}$. Let $\delta_{w}>0$ be a number that satisfies $\delta_{w}\left\|\varphi_{w}\right\| \leq \rho$. Then, for every positive integers $t$ and $\tau \leq t$ the condition $W_{\tau} / t \in B_{w, \delta_{w}}$ entails $\varphi_{w}\left(W_{\tau}-t w\right) \geq-\left\|W_{\tau}-t w\right\|\left\|\varphi_{w}\right\|>-\delta_{w}\left\|\varphi_{w}\right\| t \geq-\rho t$, namely $\varphi_{w}\left(W_{\tau}\right)-t \varphi_{w}(w)+\rho t \geq 0$. This way, bearing in mind that $\mathbb{E}\left[U_{\tau} e^{\varphi_{w}\left(W_{\tau}\right)+H_{\tau}}\right] \leq e^{z\left(\varphi_{w}\right) \tau}$ by lemma 2.5 we get for each $w \in K$ and integers $t$ and $\tau \leq t$

$$
\begin{aligned}
\mathbb{E}\left[\mathbb{1}_{\left\{\frac{W_{\tau}}{t} \in B_{w, \delta_{w}}\right\}} U_{\tau} e^{H_{\tau}}\right] & \leq \mathbb{E}\left[U_{\tau} e^{\varphi_{w}\left(W_{\tau}\right)-t \varphi_{w}(w)+t \rho+H_{\tau}}\right] \\
& \leq e^{z\left(\varphi_{w}\right) \tau-t \varphi_{w}(w)+t \rho} \\
& \leq e^{\tau\left[z\left(\varphi_{w}\right) \vee \ell_{s}\right]-t \varphi_{w}(w)+t \rho} .
\end{aligned}
$$

We also have for each $w \in K$ and $t$

$$
\mathbb{1}_{\left\{0 \in B_{w, \delta_{w}}\right\}} \leq e^{-t \varphi_{w}(w)+t \rho}
$$

because if $0 \in B_{w, \delta_{w}}$, then $\|w\|<\delta_{w}$ so that $\varphi_{w}(w) \leq \delta_{w}\left\|\varphi_{w}\right\| \leq \rho$.

Due to the compactness of $K$, there exist finitely many points $w_{1}, \ldots, w_{n}$ in $K$ such that $K \subseteq \cup_{i=1}^{n} B_{w_{i}, \delta_{w_{i}}}$. The facts that $\limsup _{t \uparrow \infty}(1 / t) \ln \mathbb{P}\left[S_{1}>t\right]=: \ell_{\mathrm{s}}$ and $z\left(\varphi_{w_{i}}\right) \vee \ell_{\mathrm{s}}>-\infty$ for each $i$ ensure the existence of a positive constant $M<+\infty$ such that for all $t$ and $i \leq n$

$$
\mathbb{P}\left[S_{1}>t\right] \leq M e^{t\left[z\left(\varphi_{w_{i}}\right) \vee \ell_{s}\right]+t \rho} .
$$

At this point, identity (3.2) combined with (3.8), (3.9), and (3.10) shows that for every $t$

$$
\begin{aligned}
\nu_{t}(K) & \leq \sum_{i=1}^{n} \mathbb{1}_{\left\{0 \in B_{w_{i}, \delta_{w_{i}}}\right\}} \cdot \mathbb{P}\left[S_{1}>t\right] \\
& +\sum_{i=1}^{n} \sum_{\tau=1}^{t} \mathbb{E}\left[\mathbb{1}_{\left\{\frac{W_{\tau}}{t} \in B_{w_{i}, \delta_{w_{i}}}\right\}} U_{\tau} e^{H_{\tau}}\right] \cdot \mathbb{P}\left[S_{1}>t-\tau\right] \\
& \leq M \sum_{i=1}^{n} \sum_{\tau=0}^{t} e^{\tau\left[z\left(\varphi_{w_{i}}\right) \vee \ell_{s}\right]-t \varphi_{w_{i}}\left(w_{i}\right)+t \rho} \cdot e^{(t-\tau)\left[z\left(\varphi_{w_{i}}\right) \vee \ell_{s}\right]+(t-\tau) \rho} \\
& \leq M \sum_{i=1}^{n} \sum_{\tau=0}^{t} e^{t\left[z\left(\varphi_{w_{i}}\right) \vee \ell_{s}\right]-t \varphi_{w_{i}}\left(w_{i}\right)+2 t \rho} \leq M n(t+1) e^{-t \lambda+2 t \rho},
\end{aligned}
$$


which in turn yields $\lim \sup _{t \uparrow \infty}(1 / t) \ln \nu_{t}(K) \leq-\lambda+2 \rho$. The proposition follows from here by sending $\rho$ to zero and $\lambda$ to $\inf _{w \in K} \sup _{\varphi \in \mathcal{X}}\left\{\varphi(w)-z(\varphi) \vee \ell_{\mathrm{s}}\right\}$.

The upper bound stated by proposition 3.2 cannot be extended in general to convex sets when $\ell_{\mathrm{s}}=-\infty$. However, at least the following weaker upper bound holds for them.

Lemma 3.1. Let $C \subseteq \mathcal{X}$ be open convex, closed convex, or any convex set in $\mathcal{B}(\mathcal{X})$ when $\mathcal{X}$ is finite-dimensional. Then, for each real number $\ell \geq \ell_{s}$

$$
\limsup _{t \uparrow \infty} \frac{1}{t} \ln \nu_{t}(C) \leq-\inf _{w \in C} \sup _{\varphi \in \mathcal{X}^{\star}}\{\varphi(w)-z(\varphi) \vee \ell\} .
$$

Proof. Pick a real number $\ell \geq \ell_{\mathrm{s}}$ and notice that $\inf _{w \in C} \sup _{\varphi \in \mathcal{X}}\{\varphi(w)-z(\varphi) \vee \ell\} \geq$ $-z(0) \vee \ell>-\infty$. Fix a real number $\lambda<\inf _{w \in C} \sup _{\varphi \in \mathcal{X}^{\star}}\{\varphi(w)-z(\varphi) \vee \ell\}$. To begin with, we observe that for any given real number $\eta \geq 1$ and integer $\tau \geq 1$ we have the bound

$$
\ln \mu_{\tau}(\eta C) \leq-\lambda \eta \tau-\ell(\eta-1) \tau,
$$

where $\eta C:=\{\eta w: w \in C\} \in \mathcal{B}(\mathcal{X})$, which is convex, open if $C$ is open, and closed if $C$ is closed. Indeed, as there exists $\epsilon>0$ such that $\lambda+\epsilon \leq \sup _{\varphi \in \mathcal{X}^{\star}}\{\varphi(w)-z(\varphi) \vee \ell\}$ for all $w \in C$, for every $w \in C$ we can find $\varphi_{w} \in \mathcal{X}^{\star}$ satisfying $\lambda \leq \varphi_{w}(w)-z\left(\varphi_{w}\right) \vee \ell$. This way, for each $w \in C$ we obtain

$$
\begin{aligned}
J(\eta w) & =\sup _{\varphi \in \mathcal{X}^{\star}}\{\varphi(\eta w)-z(\varphi)\} \geq \eta \varphi_{w}(w)-z\left(\varphi_{w}\right) \\
& \geq \eta \lambda+\eta\left[z\left(\varphi_{w}\right) \vee \ell\right]-z\left(\varphi_{w}\right) \geq \eta \lambda+(\eta-1) \ell .
\end{aligned}
$$

On the other hand, if $\gamma$ is a large enough integer so that $\gamma \tau>t_{c}$, then the convexity of $\eta C$ allows us to invoke super-additive properties to obtain $(1 / \tau) \ln \mu_{\tau}(\eta C) \leq(1 / \gamma \tau) \ln \mu_{\gamma \tau}(\eta C) \leq$ $\mathcal{L}(\eta C)$. Consequently, lemma 2.4 with the set $\eta C$ entails $\ln \mu_{\tau}(\eta C) \leq-\tau \inf _{v \in \eta C}\{J(v)\}$, which proves (3.11) because $\inf _{v \in \eta C}\{J(v)\}=\inf _{w \in C}\{J(\eta w)\} \geq \lambda \eta+\ell(\eta-1)$.

We use the bound (3.11) as follows. Given any positive integers $t$ and $\tau \leq t$, setting $\eta:=t / \tau$ we have that $W_{\tau} / \tau \in \eta C$ is tantamount to $W_{\tau} / t \in C$. This way, (3.11) yields

$$
\mathbb{E}\left[\mathbb{1}_{\left\{\frac{W_{\tau} \in C}{t} \in C\right.} U_{\tau} e^{H_{\tau}}\right]=\mu_{\tau}(\eta C) \leq e^{-\lambda t-\ell(t-\tau)} .
$$

For each $t$ we also find

$$
\mathbb{1}_{\{0 \in C\}} \leq e^{-\lambda t-\ell t}
$$

because if $0 \in C$, then $\lambda<\sup _{\varphi \in \mathcal{X}^{\star}}\{\varphi(w)-z(\varphi) \vee \ell\}$ with $w=0$ gives $\lambda \leq \sup _{\varphi \in \mathcal{X}^{\star}}\{-z(\varphi) \vee$ $\ell\} \leq-\ell$. Finally, recalling that $\lim \sup _{t \uparrow \infty}(1 / t) \ln \mathbb{P}\left[S_{1}>t\right]=: \ell_{\mathrm{s}} \leq \ell$ we realize that for any fixed number $\rho>0$ there exists a positive constant $M<+\infty$ such that $\mathbb{P}\left[S_{1}>t\right] \leq M e^{(\ell+\rho) t}$ for all $t \geq 0$. By making use of this bound in (3.2) as well as bounds (3.12) and (3.13) we find

$$
\nu_{t}(C) \leq M \sum_{\tau=0}^{t} e^{-\lambda t-\ell(t-\tau)} \cdot e^{(\ell+\rho)(t-\tau)} \leq M(t+1) e^{-\lambda t+\rho t} .
$$

Thus $\lim \sup _{t \uparrow \infty}(1 / t) \ln \nu_{t}(C) \leq-\lambda+\rho$, which proves the lemma once $\lambda$ is sent to $\inf _{w \in C} \sup _{\varphi \in \mathcal{X}^{*}}\{\varphi(w)-$ $z(\varphi) \vee \ell\}$ and $\rho$ is sent to zero.

We conclude the section demonstrating an upper large deviation bound for closed sets under the hypothesis that $\mathcal{X}$ is finite-dimensional and that an exponential moment condition holds. No restriction on $\ell_{\mathrm{s}}$ is needed here.

Proposition 3.3. Assume that $\mathcal{X}$ has finite dimension and that there exist numbers $\zeta \geq 0$ and $\xi>0$ such that $\mathbb{E}\left[e^{\xi\left\|X_{1}\right\|+v\left(S_{1}\right)-\zeta S_{1}} 1_{\left\{S_{1}<\infty\right\}}\right]<+\infty$. Then, for each $F \subseteq \mathcal{X}$ closed

$$
\limsup _{t \uparrow \infty} \frac{1}{t} \ln \nu_{t}(F) \leq-\inf _{w \in F} \sup _{\varphi \in \mathcal{X}^{\star}}\left\{\varphi(w)-z(\varphi) \vee \ell_{s}\right\} .
$$


Proof. Fix a closed set $F$ in $\mathcal{X}$ and observe that $\inf _{w \in F} \sup _{\varphi \in \mathcal{X}}\left\{\varphi(w)-z(\varphi) \vee \ell_{\mathrm{s}}\right\} \geq$ $-z(0) \vee \ell_{\mathrm{s}}>-\infty$. Pick a real number $\lambda<\inf _{w \in F} \sup _{\varphi \in \mathcal{X}^{\star}}\left\{\varphi(w)-z(\varphi) \vee \ell_{\mathrm{s}}\right\}$. Let $d$ be the dimension of $\mathcal{X}$ and let $\varphi_{1}, \ldots, \varphi_{2 d}$ be the linear functionals introduced in the proof of proposition 2.4. Since $z(\varphi)<+\infty$ if $\|\varphi\| \leq \xi$ by hypothesis, as we have seen in Section 2.4. there exists a positive number $M<+\infty$ with the property that $z\left(\xi \varphi_{i}\right) \leq M$ for each $i$. Pick a number $\rho>0$ such that $M-\xi \rho \leq-\lambda$. Denoting by $K$ the compact set $\cap_{i=1}^{2 d}\left\{w \in \mathcal{X}: \varphi_{i}(w) \leq \rho\right\}$ we have $K^{c}=\cup_{i=1}^{2 d}\left\{w \in \mathcal{X}: \varphi_{i}(w)>\rho\right\}$. This way, starting from (3.2) and noticing that $0 \notin\left\{w \in \mathcal{X}: \varphi_{i}(w)>\rho\right\}$ for all $i$, by using the Chernoff bound first and the bound $\mathbb{E}\left[U_{t} e^{\xi \varphi\left(W_{t}\right)+H_{t}}\right] \leq e^{z\left(\xi \varphi_{i}\right) t}$ due to lemma 2.5 later we obtain

$$
\begin{aligned}
\nu_{t}\left(K^{c}\right) & \leq \sum_{i=1}^{2 d} \nu_{t}\left(\left\{w \in \mathcal{X}: \varphi_{i}(w)>\rho\right\}\right) \\
& =\sum_{i=1}^{2 d} \sum_{\tau=1}^{t} \mathbb{E}\left[\mathbb{1}_{\left\{\varphi_{i}\left(W_{\tau}\right)>\rho t\right\}} U_{\tau} e^{H_{\tau}}\right] \cdot \mathbb{P}\left[S_{1}>t-\tau\right] \\
& \leq \sum_{i=1}^{2 d} \sum_{\tau=1}^{t} \mathbb{E}\left[U_{\tau} e^{\xi \varphi_{i}\left(W_{\tau}\right)-\xi \rho t+H_{\tau}}\right] \leq \sum_{i=1}^{2 d} \sum_{\tau=1}^{t} e^{z\left(\xi \varphi_{i}\right) \tau-\xi \rho t} \\
& \leq 2 d t e^{M t-\xi \rho t} \leq 2 d t e^{-\lambda t}
\end{aligned}
$$

which gives $\nu_{t}(F)=\nu_{t}(F \cap K)+\nu_{t}\left(F \cap K^{c}\right) \leq \nu_{t}(F \cap K)+2 d t e^{-\lambda t}$ for each $t$. On the other hand, proposition 3.2 with the compact set $F \cap K$ shows that $\lim \sup _{t \uparrow \infty}(1 / t) \ln \nu_{t}(F \cap$ $K) \leq-\inf _{w \in F \cap K} \sup _{\varphi \in \mathcal{X}^{\star}}\left\{\varphi(w)-z(\varphi) \vee \ell_{\mathrm{S}}\right\} \leq-\inf _{w \in F} \sup _{\varphi \in \mathcal{X}^{\star}}\left\{\varphi(w)-z(\varphi) \vee \ell_{\mathrm{S}}\right\} \leq$ $-\lambda$. Thus, $\lim \sup _{t \uparrow \infty}(1 / t) \ln \nu_{t}(F) \leq-\lambda$ and the proposition is proved by sending $\lambda$ to $\inf _{w \in F} \sup _{\varphi \in \mathcal{X} \star}\left\{\varphi(w)-z(\varphi) \vee \ell_{\mathrm{s}}\right\}$.

\subsection{Theorem 1.2 point by point and counterexamples}

Now we explicitly verify theorem 1.2 point by point. Assume $\ell_{\mathrm{s}}=-\infty$. Then, $\ell_{\mathrm{i}}=-\infty$ and starting from the facts that $\ln \mathbb{P}_{t}\left[W_{t} / t \in \cdot\right]=\ln \nu_{t}-\ln Z_{t}$ and $Z_{t}:=\mathbb{E}\left[e^{H_{t}}\right]$ for all $t \geq 1$ we get part (a) of theorem 1.2 thanks to proposition 3.1 and formula (3.4) with $\varphi=0$. Similarly, part (b) of theorem 1.2 for compact and closed sets is obtained by combining propositions 3.2 and 3.3 with formula (3.3). As far as convex sets is concerned, we observe that $z(0)-I(0)=-\sup _{\varphi \in \mathcal{X}^{\star}}\{-z(\varphi)\}=\inf _{\varphi \in \mathcal{X} \star}\{z(\varphi)\}$ so that $z(\varphi) \geq z(0)-I(0)$ for all $\varphi \in \mathcal{X}^{\star}$. This way, part (b) of theorem 1.2 for convex sets follows when $I(0)<+\infty$ by invoking lemma 3.1 with $\ell:=z(0)-I(0)$ and, again, formula (3.3) with $\varphi=0$.

The upper large deviation bound for convex sets cannot hold in general when $\ell_{\mathrm{S}}=-\infty$ and $I(0)=+\infty$. We show two examples where it fails, involving an open convex set and a closed convex set, respectively. In these examples we assume $\mathbb{P}\left[1<S_{1}<\infty\right]=1$ and $v=0$, so that $H_{t}=0, Z_{t}=1$, and $\mathbb{P}_{t}\left[W_{t} / t \in \cdot\right]=\nu_{t}$ for every $t$. The following is the counterexample with an open convex set.

Example 3.1. Consider the reward $X_{i}:=S_{i}$ for each $i$. In this example we have $\mathcal{X}=\mathbb{R}$, so that for any $\varphi \in \mathcal{X}^{\star}$ there exists one and only one real number $k$ such that $\varphi(w)=k w$ for all $w . A s \mathbb{P}\left[S_{1}<\infty\right]=1$ and $v=0$, by identifying $\varphi$ with $k$ definitions (1.1) and (1.2) give $z(k)=\inf \left\{\zeta \in \mathbb{R}: \mathbb{E}\left[e^{k S_{1}-\zeta S_{1}}\right] \leq 1\right\}=k$ for all $k \in \mathbb{R}, I(1)=0$, and $I(w)=+\infty$ for each $w \in \mathbb{R} \backslash\{1\}$. The rate function $I$ is consistent with the fact that $\sum_{i \geq 1} S_{i} \mathbb{1}_{\left\{T_{i} \leq t\right\}}=t$ if a renewal occurs at time $t$. The upper bound $\lim \sup _{t \uparrow \infty}(1 / t) \ln \mathbb{P}_{t}\left[W_{t} / t \in C\right] \leq-\inf _{w \in C}\{I(w)\}$ does not hold with the open convex set $C:=(-\infty, 1)$, for which $\inf _{w \in C}\{I(w)\}=+\infty$. Indeed, keeping only the term corresponding to $\tau=t-1$ in the r.h.s. of (3.2), observing that $W_{t-1} / t=1-1 / t \in C$ if $U_{t-1}=1$, and recalling that $\mathbb{P}\left[S_{1}>1\right]=1$ by assumption, we find for each $t \geq 2$

$$
\begin{aligned}
1 & \geq \mathbb{P}_{t}\left[\frac{W_{t}}{t} \in C\right]=\nu_{t}(C) \\
& \geq \mathbb{E}\left[\mathbb{1}_{\left\{\frac{W_{t-1}}{t} \in C\right\}} U_{t-1} e^{H_{t-1}}\right] \cdot \mathbb{P}\left[S_{1}>1\right]=\mathbb{E}\left[U_{t-1} e^{H_{t-1}}\right],
\end{aligned}
$$


giving $\lim _{t \uparrow \infty}(1 / t) \ln \mathbb{P}_{t}\left[W_{t} / t \in C\right]=0$ by lemma 2.5 as $z(0)=0$.

The following is the counterexample with a closed convex set.

Example 3.2. Consider the reward $X_{i}:=\left(S_{i}, Y_{i}\right)$ for each $i$ with $Y_{i}$ independent of $S_{i}$ and distributed according to the standard Cauchy law: $\mathbb{P}\left[Y_{i} \leq y\right]=(1 / \pi)[\pi / 2+\arctan (y)]$ for all $y \in \mathbb{R}$. In this example $\mathcal{X}=\mathbb{R}^{2}$, so that for any $\varphi \in \mathcal{X}^{\star}$ there exists one and only one pair of real numbers $k=\left(k_{S}, k_{Y}\right)$ such that $\varphi(w)=k_{S} w_{S}+k_{Y} w_{Y}$ for all $w=\left(w_{S}, w_{Y}\right)$. As $\mathbb{P}\left[S_{1}<\infty\right]=1$ and $v=0$, and as $Y_{1}$ has no exponential moments, by identifying $\varphi$ with $k$ definition (1.1) gives $z(k)=\inf \left\{\zeta \in \mathbb{R}: \mathbb{E}\left[e^{k_{S} S_{1}-\zeta S_{1}}\right] \cdot \mathbb{E}\left[e^{k_{Y} Y_{1}}\right] \leq 1\right\}=k_{S}$ if $k_{Y}=0$ and $z(k)=+\infty$ if $k_{Y} \neq 0$. It follows from definition (1.2) that $I(w)=0$ if $w_{S}=1$ and $I(w)=+\infty$ otherwise. The upper bound $\lim \sup _{t \uparrow \infty}(1 / t) \ln \mathbb{P}_{t}\left[W_{t} / t \in C\right] \leq-\inf _{w \in C}\{I(w)\}$ does not hold with the closed convex set $C:=\left\{w \in \mathbb{R}^{2}: w_{S}<1\right.$ and $\left.w_{Y} \geq 1 /\left(1-w_{S}\right)\right\}$, for which $\inf _{w \in C}\{I(w)\}=+\infty$. Indeed, as we shall show in a moment we have for every $t \geq 2$

$$
1 \geq \mathbb{P}_{t}\left[\frac{W_{t}}{t} \in C\right]=\nu_{t}(C) \geq \mathbb{P}\left[Y_{1} \geq t^{2}\right] \cdot \mathbb{E}\left[U_{t-1} e^{H_{t-1}}\right]
$$

giving $\lim _{t \uparrow \infty}(1 / t) \ln \mathbb{P}_{t}\left[W_{t} / t \in C\right]=0$ by lemma 2.5 as $z(0)=0$.

In order to prove (3.14) we pick an integer $t \geq 2$ and observe that when a renewal occurs at the time $t-1$, so that $\sum_{i \geq 1} S_{i} \mathbb{1}_{\left\{T_{i} \leq t-1\right\}}=t-1$, then $W_{t-1} / t \in C$ if and only if $\sum_{i \geq 1} Y_{i} \mathbb{1}_{\left\{T_{i} \leq t-1\right\}} \geq t^{2}$. This way, keeping only the term corresponding to $\tau=t-1$ in the r.h.s. of (3.2) and recalling that $\mathbb{P}\left[S_{1}>1\right]=1$ we get

$$
\begin{aligned}
\nu_{t}(C) & \geq \mathbb{E}\left[\mathbb{1}_{\left\{\frac{\left.W_{t-1} \in C\right\}}{t} \in U_{t-1} e^{H_{t-1}}\right] \cdot \mathbb{P}\left[S_{1}>1\right]}\right. \\
& =\mathbb{E}\left[\mathbb{1}_{\left\{\sum_{i \geq 1} Y_{i} \mathbb{1}_{\left\{T_{i} \leq t-1\right\}} \geq t^{2}\right\}} U_{t-1} e^{H_{t-1}}\right] \\
& =\sum_{n=1}^{t-1} \mathbb{E}\left[\mathbb{1}_{\left\{\sum_{i=1}^{n} Y_{i} \geq t^{2}\right\}} \mathbb{1}_{\left\{T_{n}=t-1\right\}} e^{H_{t-1}}\right] \\
& =\sum_{n=1}^{t-1} \mathbb{P}\left[\sum_{i=1}^{n} Y_{i} \geq t^{2}\right] \cdot \mathbb{E}\left[\mathbb{1}_{\left\{T_{n}=t-1\right\}} e^{H_{t-1}}\right] .
\end{aligned}
$$

On the other hand, $(1 / n) \sum_{i=1}^{n} Y_{i}$ is distributed as $Y_{1}$ by the stability property of the Cauchy law so that

$$
\begin{aligned}
\nu_{t}(C) & \geq \sum_{n=1}^{t-1} \mathbb{P}\left[\sum_{i=1}^{n} Y_{i} \geq t^{2}\right] \cdot \mathbb{E}\left[\mathbb{1}_{\left\{T_{n}=t-1\right\}} e^{H_{t-1}}\right] \\
& =\sum_{n=1}^{t-1} \mathbb{P}\left[n Y_{1} \geq t^{2}\right] \cdot \mathbb{E}\left[\mathbb{1}_{\left\{T_{n}=t-1\right\}} e^{H_{t-1}}\right] \\
& \geq \sum_{n=1}^{t-1} \mathbb{P}\left[Y_{1} \geq t^{2}\right] \cdot \mathbb{E}\left[\mathbb{1}_{\left\{T_{n}=t-1\right\}} e^{H_{t-1}}\right] \\
& =\mathbb{P}\left[Y_{1} \geq t^{2}\right] \cdot \mathbb{E}\left[U_{t-1} e^{H_{t-1}}\right] .
\end{aligned}
$$

\subsection{Theorem 1.3 point by point}

To conclude, we explicitly verify theorem 1.3 point by point. Assume $\ell_{\mathrm{s}}>-\infty$. The functions $I_{\mathrm{i}}$ and $I_{\mathrm{S}}$ defined by (1.3) and (1.4) are the Fenchel-Legendre transform of $z \vee \ell_{\mathrm{i}}-$ $z(0) \vee \ell_{\mathrm{S}}$ and $z \vee \ell_{\mathrm{S}}-z(0) \vee \ell_{\mathrm{i}}$, respectively. Convexity and lower semicontinuity of $I_{\mathrm{i}}$ and $I_{\mathrm{S}}$ are immediate to check. The functions $I_{\mathrm{i}}$ and $I_{\mathrm{s}}$ are proper convex. Indeed, considering for instance $I_{\mathrm{i}}$, we have on the one hand $I_{\mathrm{i}}(w) \geq-z(0) \vee \ell_{\mathrm{i}}+z(0) \vee \ell_{\mathrm{s}}>-\infty$ for all $w \in \mathcal{X}$, and on the other hand $I_{\mathrm{i}}(u) \leq J(u)+z(0) \vee \ell_{\mathrm{s}}<+\infty$ at some point $u$ because $J$ is proper convex. These arguments demonstrate part (a) of theorem 1.3. As far as part (b) and part (c) is 
concerned, we recall that $\ln \mathbb{P}_{t}\left[W_{t} / t \in \cdot\right]=\ln \nu_{t}-\ln Z_{t}$ and that $Z_{t}:=\mathbb{E}\left[e^{H_{t}}\right]$ for all $t$ in such a way that part (b) follows from proposition 3.1 and formula (3.4) with $\varphi=0$. Part (c) for compact and closed sets is due to propositions 3.2 and 3.3 combined with formula (3.3). Finally, part (c) for convex sets follows from lemma 3.1 with $\ell=\ell_{\mathrm{s}}$ and, again, formula (3.3).

\section{Acknowledgements}

The author is grateful to Paolo Tilli for useful discussions about the counterexamples presented in Section 3.3 and to Francesco Caravenna and Paolo Dai Pra for valuable overall comments.

\section{A Proof of lemma 1.1}

Since $S_{1}<\infty$ with full probability and $v=0$, according to definition (1.1) we have $z(\varphi)=$ $\inf \left\{\zeta \in \mathbb{R}: \mathbb{E}\left[e^{\varphi\left(X_{1}\right)-\zeta S_{1}}\right] \leq 1\right\}$ for all $\varphi \in \mathcal{X}^{\star}$. We shall show that for every $\beta \geq 0$ and $w \in \mathcal{X}$

$$
\Upsilon(\beta, w)=\sup _{\varphi \in \operatorname{dom} z}\{\varphi(w)-\beta z(\varphi)\}
$$

where $\operatorname{dom} z:=\left\{\varphi \in \mathcal{X}^{\star}: z(\varphi)<+\infty\right\}$ is the effective domain of the function $z$. The identity $I=\Lambda$ immediately follows from (A.1) by taking $\beta=1$ and proves part (a) of the lemma. Regarding part (b), assume $\ell_{\mathrm{s}}>-\infty$ and consider the function $F$ that for a given $w \in \mathcal{X}$ maps $(\beta, \varphi) \in[0,1] \times \operatorname{dom} z$ in the real number $F(\beta, \varphi):=\varphi(w)-\beta z(\varphi)-(1-\beta) \ell_{\mathrm{s}}$. The function $F$ is concave and upper semicontinuous with respect to $\varphi$ for each fixed $\beta \in$ $[0,1]$, inheriting these properties from $z$, and convex and continuous with respect to $\beta$ for each fixed $\varphi \in \operatorname{dom} z$. Then, due to compactness of the closed interval $[0,1]$, Sion's minimax theorem allows us to exchange the infimum over $\beta \in[0,1]$ and the supremum over $\varphi \in \operatorname{dom} z: \sup _{\varphi \in \operatorname{dom} z} \inf _{\beta \in[0,1]}\{F(\beta, \varphi)\}=\inf _{\beta \in[0,1]} \sup _{\varphi \in \operatorname{dom} z}\{F(\beta, \varphi)\}$. Since $z(0)=0$ and $z(\varphi)=+\infty$ if $\varphi \notin \operatorname{dom} z$, this identity yields

$$
\begin{aligned}
I_{\mathrm{S}}(w) & =\sup _{\varphi \in \mathcal{X}^{\star}}\left\{\varphi(w)-z(\varphi) \vee \ell_{\mathrm{s}}\right\}=\sup _{\varphi \in \operatorname{dom} z} \inf _{\beta \in[0,1]}\{F(\beta, \varphi)\} \\
& =\inf _{\beta \in[0,1]} \sup _{\varphi \in \operatorname{dom} z}\{F(\beta, \varphi)\}=\inf _{\beta \in[0,1]} \sup _{\varphi \in \operatorname{dom} z}\left\{\varphi(w)-\beta z(\varphi)-(1-\beta) \ell_{\mathrm{s}}\right\} \\
& =\inf _{\beta \in[0,1]}\left\{\Upsilon(\beta, w)-(1-\beta) \ell_{\mathrm{s}}\right\}=: \Lambda_{\mathrm{S}}(w) .
\end{aligned}
$$

This way, part (b) of the lemma is demonstrated as $w$ is an arbitrary point.

Let us prove (A.1). Pick $\beta \geq 0$ and $w \in \mathcal{X}$. To begin with, we point out that the function that associates $\zeta$ with $\mathbb{E}\left[e^{\varphi\left(X_{1}\right)-\zeta S_{1}}\right]$ for a given $\varphi \in \mathcal{X}^{\star}$ is lower semicontinuous by Fatou's lemma, so that $\mathbb{E}\left[e^{\varphi\left(X_{1}\right)-z(\varphi) S_{1}}\right] \leq 1$ if $z(\varphi)<+\infty$. It follows that for any $\varphi \in \operatorname{dom} z$

$$
\begin{aligned}
\Upsilon_{\mathrm{C}}(\beta, w) & :=\sup _{(\zeta, \vartheta) \in \mathbb{R} \times \mathcal{X}^{\star}}\left\{\vartheta(w)-\beta \zeta-\ln \mathbb{E}\left[e^{\vartheta\left(X_{1}\right)-\zeta S_{1}}\right]\right\} \\
& \geq \varphi(w)-\beta z(\varphi)-\ln \mathbb{E}\left[e^{\varphi\left(X_{1}\right)-z(\varphi) S_{1}}\right] \geq \varphi(w)-\beta z(\varphi),
\end{aligned}
$$

so that $\inf _{\gamma>0}\left\{\gamma \Upsilon_{\mathrm{C}}(\beta / \gamma, w / \gamma)\right\} \geq \varphi(w)-\beta z(\varphi)$. Continuity of $\varphi$ results in $\Upsilon(\beta, w) \geq$ $\varphi(w)-\beta z(\varphi)$ and the arbitrariness of $\varphi$ gives

$$
\Upsilon(\beta, w) \geq \sup _{\varphi \in \operatorname{dom} z}\{\varphi(w)-\beta z(\varphi)\}
$$

The opposite bound, which leads us to the proof of (A.1), is more involved and is achieved through the following two inequalities:

$$
\begin{aligned}
\Upsilon(\beta, w) & \leq \inf _{\gamma \in[0, \beta]} \sup _{(\zeta, \varphi) \in \mathcal{D}}\left\{\varphi(w)-\beta \zeta-\gamma \ln \mathbb{E}\left[e^{\varphi\left(X_{1}\right)-\zeta S_{1}}\right]\right\} \\
& \leq \sup _{\varphi \in \operatorname{dom} z}\{\varphi(w)-\beta z(\varphi)\}
\end{aligned}
$$


where $\mathcal{D}:=\left\{(\zeta, \varphi) \in \mathbb{R} \times \mathcal{X}^{\star}: \mathbb{E}\left[e^{\varphi\left(X_{1}\right)-\zeta S_{1}}\right]<+\infty\right\}$. To get at (A.2) we observe that the definition of $\Upsilon$ immediately gives

$$
\Upsilon(\beta, w) \leq \sup _{(\zeta, \varphi) \in \mathcal{D}}\left\{\varphi(w)-\beta \zeta-\gamma \ln \mathbb{E}\left[e^{\varphi\left(X_{1}\right)-\zeta S_{1}}\right]\right\}
$$

for all $\gamma>0$. The lower-semicontinuous regularization procedure used to construct $\Upsilon$ entails that this bound also holds for $\gamma=0$, as we now show, thus giving (A.2). The rate function $\Upsilon_{\mathrm{C}}$ is proper convex by Cramér's theorem, so that there exist $\beta_{o} \in \mathbb{R}$ and $w_{o} \in \mathcal{X}$ such that $\Upsilon_{\mathrm{C}}\left(\beta_{o}, w_{o}\right)$ is finite. It follows that $\varphi\left(w_{o}\right)-\beta_{o} \zeta-\ln \mathbb{E}\left[e^{\varphi\left(X_{1}\right)-\zeta S_{1}}\right] \leq \Upsilon_{\mathrm{C}}\left(\beta_{o}, w_{o}\right)=: c \geq 0$, that is $\ln \mathbb{E}\left[e^{\varphi\left(X_{1}\right)-\zeta S_{1}}\right] \geq \varphi\left(w_{o}\right)-\beta_{o} \zeta-c$ for all $\zeta \in \mathbb{R}$ and $\varphi \in \mathcal{X}^{\star}$. Then, for every $\delta>0$ and $\gamma_{o} \in(0, \delta)$ such that $\gamma_{o}\left|\beta_{o}\right|<\delta$ and $\gamma_{o}\left\|w_{o}\right\|<\delta$ we find

$$
\begin{aligned}
\inf _{\alpha \in(\beta-\delta, \beta+\delta)} \inf _{u \in B_{w, \delta}} \inf _{\gamma>0}\left\{\gamma \Upsilon_{\mathrm{C}}(\alpha / \gamma, u / \gamma)\right\} & \leq \inf _{\gamma>0}\left\{\gamma \Upsilon_{\mathrm{C}}\left(\beta / \gamma+\gamma_{o} \beta_{o} / \gamma, w / \gamma+\gamma_{o} w_{o} / \gamma\right)\right\} \\
& \leq \gamma_{o} \Upsilon_{\mathrm{C}}\left(\beta / \gamma_{o}+\beta_{o}, w / \gamma_{o}+w_{o}\right) \\
& =\sup _{(\varphi, \zeta) \in \mathcal{D}}\left\{\varphi\left(w+\gamma_{o} w_{o}\right)-\left(\beta+\gamma_{o} \beta_{o}\right) \zeta-\gamma_{o} \ln \mathbb{E}\left[e^{\varphi\left(X_{1}\right)-\zeta S_{1}}\right]\right\} \\
& \leq \sup _{(\varphi, \zeta) \in \mathcal{D}}\{\varphi(w)-\beta \zeta\}+\gamma_{o} c \\
& \leq \sup _{(\varphi, \zeta) \in \mathcal{D}}\{\varphi(w)-\beta \zeta\}+\delta c .
\end{aligned}
$$

It follows that

$$
\begin{aligned}
\Upsilon(\beta, w) & :=\lim _{\delta \downarrow 0} \inf _{\alpha \in(\beta-\delta, \beta+\delta)} \inf _{u \in B_{w, \delta}} \inf _{\gamma>0}\left\{\gamma \Upsilon_{\mathrm{C}}(\alpha / \gamma, u / \gamma)\right\} \\
& \leq \sup _{(\varphi, \zeta) \in \mathcal{D}}\{\varphi(w)-\beta \zeta\}
\end{aligned}
$$

which exactly is (A.4) when $\gamma=0$.

Let us move to bound (A.3), which we prove by invoking Sion's minimax theorem once again. The function that associates $(\zeta, \varphi) \in \mathcal{D}$ with $\mathbb{E}\left[e^{\varphi\left(X_{1}\right)-\zeta S_{1}}\right]$ is lower semicontinuous by Fatou's lemma and convex, so that the real function that maps $(\gamma, \zeta, \varphi) \in[0, \beta] \times \mathcal{D}$ in $\varphi(w)-\beta \zeta-\gamma \ln \mathbb{E}\left[e^{\varphi\left(X_{1}\right)-\zeta S_{1}}\right]$ is concave and upper semicontinuous with respect to $(\zeta, \varphi)$ for each fixed $\gamma \in[0, \beta]$ and convex and continuous with respect to $\gamma$ for each fixed pair $(\zeta, \varphi) \in \mathcal{D}$. Then, Sion's theorem ensures us that

$$
\begin{aligned}
& \inf _{\gamma \in[0, \beta]} \sup _{(\zeta, \varphi) \in \mathcal{D}}\left\{\varphi(w)-\beta \zeta-\gamma \ln \mathbb{E}\left[e^{\varphi\left(X_{1}\right)-\zeta S_{1}}\right]\right\} \\
= & \sup _{(\zeta, \varphi) \in \mathcal{D}} \inf _{\gamma \in[0, \beta]}\left\{\varphi(w)-\beta \zeta-\gamma \ln \mathbb{E}\left[e^{\varphi\left(X_{1}\right)-\zeta S_{1}}\right]\right\} \\
= & \sup _{(\zeta, \varphi) \in \mathcal{D}}\left\{\varphi(w)-\beta \zeta-\beta \ln 1 \vee \mathbb{E}\left[e^{\varphi\left(X_{1}\right)-\zeta S_{1}}\right]\right\} .
\end{aligned}
$$

On the other hand, if $(\zeta, \varphi) \in \mathcal{D}$, then $\varphi \in \operatorname{dom} z$ because $\mathbb{E}\left[e^{\varphi\left(X_{1}\right)-(\zeta+h) S_{1}}\right] \leq e^{-h} \mathbb{E}\left[e^{\varphi\left(X_{1}\right)-\zeta S_{1}}\right] \leq$ 1 for all sufficiently large $h$ as $S_{1} \geq 1$ with full probability. It follows that

$$
\begin{aligned}
& \inf _{\gamma \in[0, \beta]} \sup _{(\zeta, \varphi) \in \mathcal{D}}\left\{\varphi(w)-\beta \zeta-\gamma \ln \mathbb{E}\left[e^{\varphi\left(X_{1}\right)-\zeta S_{1}}\right]\right\} \\
= & \sup _{(\zeta, \varphi) \in \mathcal{D}}\left\{\varphi(w)-\beta \zeta-\beta \ln 1 \vee \mathbb{E}\left[e^{\varphi\left(X_{1}\right)-\zeta S_{1}}\right]\right\} \\
\leq & \sup _{\varphi \in \operatorname{dom}} \sup _{\zeta \in \mathbb{R}}\left\{\varphi(w)-\beta \zeta-\beta \ln 1 \vee \mathbb{E}\left[e^{\varphi\left(X_{1}\right)-\zeta S_{1}}\right]\right\} \\
\leq & \sup _{\varphi \in \operatorname{dom} z}\{\varphi(w)-\beta z(\varphi)\},
\end{aligned}
$$

where the last bound demonstrates (A.3) and is justified as follows. Pick any $\varphi \in \operatorname{dom} z$ and recall that $\mathbb{E}\left[e^{\varphi\left(X_{1}\right)-\zeta S_{1}}\right] \leq 1$ or $\mathbb{E}\left[e^{\varphi\left(X_{1}\right)-\zeta S_{1}}\right]>1$ depending on whether $\zeta \geq z(\varphi)$ or 
$\zeta<z(\varphi)$ by definition of $z(\varphi)$. The function that associates $\zeta$ with $\zeta+\ln 1 \vee \mathbb{E}\left[e^{\varphi\left(X_{1}\right)-\zeta S_{1}}\right]$ is lower semicontinuous and increasing for $\zeta \geq z(\varphi)$. It is not increasing for $\zeta<z(\varphi)$ because $\zeta+\ln 1 \vee \mathbb{E}\left[e^{\varphi\left(X_{1}\right)-\zeta S_{1}}\right]=\ln \mathbb{E}\left[e^{\varphi\left(X_{1}\right)-\zeta\left(S_{1}-1\right)}\right]$ in this case and $S_{1} \geq 1$ with full probability. Then, this function attains a global minimum at $\zeta=z(\varphi)$, so that $\zeta+\ln 1 \vee \mathbb{E}\left[e^{\varphi\left(X_{1}\right)-\zeta S_{1}}\right] \geq$ $z(\varphi)$ for all $\zeta \in \mathbb{R}$.

\section{References}

[1] S. Asmussen, Applied Probability and Queues, 2nd ed., Springer, New York, 2003.

[2] D.C.M. Dickson, Insurance Risk and Ruin, 2nd ed., Cambridge University Press, Cambridge, 2017.

[3] T. Rolski, H. Schmidli, V. Schmidt, J.L. Teugels, Stochastic Processes for Insurance and Finance, Wiley, Chichester, 1999.

[4] M. Ledoux, M. Talagrand, Probability in Banach Spaces, Springer, Berlin, 1991.

[5] R.R. Bahadur, S. Zabell, Large deviations of the sample mean in general vector spaces, Ann. Probab. 7 (1979) 587-621.

[6] M.D. Donsker, S.R.S. Varadhan, Asymptotic evaluation of certain Markov process expectations for large time - III, Commun. Pure Appl. Math. 29 (1976) 389-461.

[7] G. Giacomin, Random Polymer Models, Imperial College Press, London, 2007.

[8] F. den Hollander, Random Polymers, Springer, Berlin, 2009.

[9] D. Ruelle, Correlation functionals, J. Math. Physics 6 (1965) 201-220.

[10] O.E. Lanford, Entropy and equilibrium states in classical statistical mechanics, In Statistical Mechanics and Mathematical Problems. Lecture Notes in Physics 20 1-113, Springer, Berlin (1971).

[11] M. Zamparo, Large deviations in renewal models of statistical mechanics, J. Phys. A 52 (2019) 495004.

[12] A. Dembo, O. Zeitouni, Large Deviations Techniques and Applications, 2nd ed., Springer, New York, 1998.

[13] F. den Hollander, Large Deviations, American Mathematical Society, Providence, 2000.

[14] P.W. Glynn, W. Whitt, Large deviations behavior of counting processes and their inverses, Queueing Syst. Theory Appl. 17 (1994) 107-128.

[15] C. Macci, Large deviation results for compound Markov renewal processes, Braz. J. Probab. Stat. 19 (2005) 1-12.

[16] C. Macci, Large deviations for compound Markov renewal processes with dependent jump sizes and jump waiting times, Bull. Belg. Math. Soc. Simon Stevin 14 (2007) 213-228.

[17] A.A. Puhalskii, W. Whitt, Functional large deviation principles for first-passage-time processes, Ann. Appl. Probab. 7 (1997) 362-381.

[18] N.G. Duffield, W. Whitt, Large deviations of inverse processes with nonlinear scalings, Ann. Appl. Probab. 8 (1998) 995-1026.

[19] K. Duffy and M. Rodgers-Lee, Some useful functions for functional large deviations, Stoch. Stoch. Rep. 76 (2004) 267-279.

[20] T. Jiang, Large deviations for renewal processess, Stochastic Process. Appl. 50 (1994) 57-71. 
[21] A.A. Borovkov, A.A. Mogulskii, Large deviation principles for the finite-dimensional distributions of compound renewal processes, Sib. Math. J. 56 (2015) 28-53.

[22] A.A. Borovkov, On large deviation principles for compound renewal processes, Math. Notes 106 (2019) 864-871.

[23] A.A. Borovkov, A.A. Mogulskii, Large deviation principles for trajectories of compound renewal processes. I, Theory Probab. Appl. 60 (2016) 207-224.

[24] A.A. Borovkov, A.A. Mogulskii, Large deviation principles for trajectories of compound renewal processes. II, Theory Probab. Appl. 60 (2016) 349-366.

[25] A.A. Borovkov, Large deviation principles in boundary problems for compound renewal processes, Sib. Math. J. 57 (2016) 442-469.

[26] R. Lefevere, M. Mariani, L. Zambotti, Large deviations for renewal processes, Stochastic Process. Appl. 121 (2011) 2243-2271.

[27] M. Mariani, L. Zambotti, A renewal version of the Sanov theorem, Electron. Commun. Probab. 19 (2014) 1-13.

[28] A. Schied, Cramer's condition and Sanov's theorem, Stat. Probab. Lett. 39 (1998) 55-60.

[29] B. Tsirelson, From uniform renewal theorem to uniform large and moderate deviations for renewal-reward processes, Electron. Commun. Probab. 18 (2013) 1-13.

[30] R.F. Serfozo, Large deviations of renewal processes, Stochastic Process. Appl. 2 (1974) 295-301.

[31] T. Kuczek, K.N. Crank, A large-deviation result for regenerative processes, J. Theoret. Probab. 4 (1991) 551-561.

[32] Z. Chi, Uniform convergence of exact large deviations for renewal-reward processes, Ann. Appl. Probab. 17 (2007) 1019-1048.

[33] A.A. Borovkov, A.A. Mogulskii, Integro-local limit theorems for compound renewal processes under Cramér condition. I, Sib. Math. J. 59 (2018) 383-402.

[34] A.A. Borovkov, A.A. Mogulskii, Integro-local limit theorems for compound renewal processes under Cramér condition. II, Sib. Math. J. 59 (2018) 578-597.

[35] R.S. Ellis, Entropy, Large Deviations, and Statistical Mechanics, Springer, New York, 1985.

[36] M. Costeniuc, R.S. Ellis, H. Touchette, Complete analysis of phase transitions and ensemble equivalence for the Curie-Weiss-Potts model, J. Math. Phys. 46 (2005) 063301.

[37] R.S. Ellis, P.T. Otto, H. Touchette, Analysis of phase transitions in the mean-field Blume-Emery-Griffiths model, Ann. Appl. Prob. 15 (2005) 2203-2254.

[38] R.S. Ellis, An overview of the theory of large deviations and applications to statistical mechanics, Scand. Actuarial J. No. 1 (1995) 97-142.

[39] H. Föllmer, S. Orey, Large deviations for the empirical field of a Gibbs measure, Ann. Probab. 16 (1987) 961-977.

[40] S. Olla, Large deviations for Gibbs random fields, Probab. Th. Rel. Fields 77 (1988) 343-357.

[41] H.-O. Georgii, Large deviations and maximum entropy principle for interacting random fields on $\mathbb{Z}^{d}$, Ann. Probab. 21 (1993) 1845-1875.

[42] R. Lefevere, M. Mariani, L. Zambotti, Large deviations of the current in stochastic collisional dynamics, J. Math. Phys. 52 (2011) 033302. 
[43] R. Lefevere, M. Mariani, L. Zambotti, Large deviations for a random speed particle, ALEA, Lat. Am. J. Probab. Math. Stat. 9 (2012) 739-760.

[44] V.I. Bogachev, Measure Theory, Vol. II, Springer, Berlin, 2007.

[45] W. Rudin, Functional Analysis, 2nd ed., McGraw-Hill, New York, 1991.

[46] C. Zălinescu, Convex Analysis in General Vector Spaces, World Sciencetific Publishing, Singapore, 2002.

[47] R. Cerf, P. Petit, A short proof of Cramér's Theorem in $\mathbb{R}$, Amer. Math. Monthly 118 (2011) 925-931. 